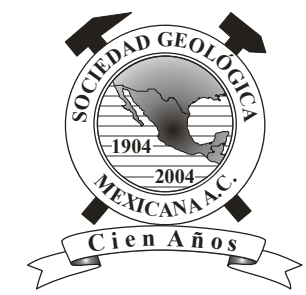

\title{
La vida temprana en la Tierra y los primeros ecosistemas terrestres
}

\author{
Hugo Beraldi-Campesi $i^{1, *}$ \\ ${ }^{1}$ Instituto de Geología, UNAM., Ciudad Universitaria, 04510, México D.F. \\ *hberaldi@unam.mx
}

\begin{abstract}
Resumen
Los ecosistemas terrestres han sido considerados tradicionalmente como superficies dominadas por plantas, cuyos primeros registros datan del Fanerozoico temprano ( $<550 \mathrm{Ma}$ /millones de años). Sin embargo, la presencia de componentes biológicos mucho más antiguos que las plantas en hábitats tan distintos como suelos, turberas, estanques, lagos, arroyos y dunas, sugiere que los ecosistemas terrestres comenzaron a existir en la Tierra hace al menos 2700 Ma. Los microbios fueron abundantes hace $~ 3500$ Ma y sin duda se adaptaron a vivir en condiciones subaéreas, en entornos intermareales y en zonas áridas y semiáridas, como lo hacen actualmente los microbios terrestres, y que tienen una enorme y rápida capacidad de adaptación a condiciones cambiantes. Todo ello está respaldado por el registro fósil. No obstante, esta evidencia es inusual e indirecta en comparación con fósiles de ambientes marinos, superficiales o profundos, y su registro ha sido poco atendido. En consecuencia, la noción de que fueron comunidades microbianas las que formaron los primeros ecosistemas terrestres no ha sido ampliamente difundido ni incorporado conceptualmente en la sociedad. Hoy conocemos un amplio registro fósil de biota marina somera y lacustre a partir de los $\sim 3500 \mathrm{Ma}$, así como microbios colonizando ambientes costeros desde hace $\sim 3450$ Ma y evidencia indirecta de actividad biológica en paleosuelos de $>3400$ Ma de edad. El tipo de ambientes, de donde proviene esta evidencia, sugiere que la vida terrestre se produjo casi en paralelo con la vida acuática en el Arqueano. Las rápidas adaptaciones observadas en microbios actuales, su excepcional tolerancia a condiciones extremas y fluctuantes, su rápida y temprana diversificación y su antiguo registro fósil, indican que los primeros ecosistemas terrestres fueron exclusivamente microbianos. Es factible que los microbios contribuyeran en la formación de los primeros suelos donde las plantas se desarrollaron más tarde. Comprender cómo la vida se diversificó y adaptó a las condiciones terrestres es fundamental para entender su impacto en los sistemas terrestres durante millones de años.
\end{abstract}

Palabras clave: Ecosistemas terrestres primitivos, cianobacterias.

\begin{abstract}
Terrestrial ecosystems have been largely regarded as plant-dominated land surfaces, with the earliest records appearing in the early Phanerozoic $(<550 \mathrm{Ma})$. However, the presence of biological components much older than plants, in habitats as different as soils, peats, ponds, lakes, streams, and dune fields, suggests that much earlier types of terrestrial ecosystems appeared in Earth at least 2700 Ma ago. Microbes were abundant $3500 \mathrm{Ma}$ ago, and they surely adapted to live in subaerial conditions in peritidal and in arid and semiarid environments, as presently done by terrestrial microbes, which have great and rapid capacity of adapting themselves to changing conditions as suggested by fossil records. Yet, this evidence is rare and indirect in comparison with fossils from shallow or deeper marine environments, and its record has been largely overlooked. Consequently, the notion that microbial communities may
\end{abstract}

Versión castellana, bajo licencia del editor de la revista, del artículo "Early life on land and the first terrestrial ecosystems" del mismo autor. This paper was published in the journal Ecological Processes, Volume 2, Thematic series 'Biological soil crusts: their diversity, functional ecology and management', edited by Bettina Weber and Jayne Belnap (2013). It can be accessed at $<<$ http://www.ecologicalprocesses.com/series/BSC $>>$. Copyright $\mathbb{C}$ 2013, Springer Open Journals. All rights reserved. 
have formed the earliest land ecosystems has not been widely accepted nor integrated into our general knowledge. Nowadays, an ample record of shallow-marine and lacustrine biota in 3500 Ma-old deposits is known, together with evidence of microbial colonization of coastal environments $\sim 3450 \mathrm{Ma}$ ago, and indirect evidence that suggest biological activity in > 3400 Ma-old paleosols. The type of ambiances from where this evidence derives endorses the idea that life on land perhaps occurred in parallel with aquatic life back in the Archean. The rapid adaptations seen in modern microbes, their outstanding tolerance to extreme and fluctuating conditions, their early and rapid diversification, and their old fossil record, collectively suggest that they constituted the earliest terrestrial ecosystems. It is likely that microbes contributed in forming the biomass-rich cover where plants later evolved. Understanding how life diversified and adapted to terrestrial conditions is critical to comprehend its impact on the Earth's systems over millions of years.

Keywords: Primitive terrestrial ecosystems, cyanobacteria.

\section{Introducción}

\subsection{Definición de terrestre}

Los ambientes terrestres seguramente han existido a través de toda la historia geológica de la Tierra a menos que su superficie estuviera permanentemente bajo el agua, lo cual no es aceptablemente realista. La definición de 'terrestre' no es tan trivial como parece. Aunque aquí se define como un ambiente 'no acuático', la distinción entre lo marino y lo terrestre atraviesa un amplio espectro de ambientes transicionales, donde lo acuático y lo no-acuático evolucionan y superponen en el tiempo. Comúnmente se entiende que un entorno terrestre (sobre el nivel del mar), puede incluir ambientes acuáticos (lagos cubiertos o no de hielo, lagunas y humedales, turberas, ríos y arroyos, campos geotérmicos) y no-acuáticos (especialmente zonas con poca lluvia). Estos hábitats pueden experimentar cambios rápidos y lentos, dependiendo de la actividad tectónica y las condiciones climáticas existentes, incluyendo la subida y bajada del nivel del mar, las glaciaciones, y la precipitación (i.e. Romans y Graham, 2013). Estos factores, a su vez influyen en la topografía regional, en las tasas de sedimentación, y en la erosión y dinámica sedimentaria. Esta combinación de factores dificulta, a veces, la interpretación de los diferentes paleoambientes representados en las rocas, en cuanto a la distinción entre un hábitat totalmente subacuático y uno completamente subaéreo. Por ejemplo, las zonas costeras muestran una gran diversidad de ambientes (deltas, estuarios, lagunas, zonas evaporíticas, dunas, etc.) que pueden cambiar su configuración y dinámica sedimentaria en tiempos relativamente cortos (de días a décadas; i.e. Hamblin y Christensen, 2007), debido a cambios en el nivel del mar. De esa manera, en pocos cientos o miles de años, una zona costera puede quedar bajo el agua o completamente expuesta a la erosión subaérea. Reconocer episodios aislados pero importantes en unos pocos centímetros o metros de estratos, así como conceptualizar los periodos de tiempo que representan en las rocas, no siempre se cumple y con frecuencia se pasa por alto en estudios regionales de menor resolución.

Es también posible que los depósitos sedimentarios originados en entornos totalmente acuáticos (fluviales, lacustres, marinos poco profundos) sean eventualmente expuestos a la atmósfera durante largos períodos de tiempo y experimenten procesos pedogenéticos que transforman el depósito original en suelo (i.e. Paul et al., $2001 \mathrm{y}$ referencias incluidas). De este modo, las rocas mantienen características primarias del depósito original, pero con características secundarias superpuestas, derivadas de condiciones ambientales in situ muy distintas a las primeras. En este sentido, el estudio de los procesos pedogenéticos (i.e. desarrollo de horizontes, capas duras o duricrusts, peds y arcillas, slickensides, etc.), así como los depósitos hidrotermales y fluviales (i.e. travertinos, tufas, sínters) y hábitats microbianos superficiales (hábitats endolíticos $\mathrm{y}$ cubiertas criptogámicas), es de particular importancia para una mejor comprensión de la vida continental en el pasado, debido a que representan hábitats terrestres y subaéreos, esperados en antiguas superficies continentales. En suma, la distinción entre los depósitos terrestres y acuáticos en el registro geológico es fundamental, más no trivial, para la comprensión de los ecosistemas terrestres primitivos y sus habitantes.

\subsection{Precaución y re-interpretación del registro geológico}

A través del estudio integral de las rocas y la comprensión de los procesos que las formaron, incluyendo el estudio del registro fósil y de la capacidad de fechar materiales, se ha desarrollado un concepto sobre cómo han evolucionado la geósfera y la biósfera a través del tiempo (ver compendios de Schopf, 1983; Canup y Righter, 2000; Eriksson et al., 2004; Schieber et al., 2007; van Kranendonk et al., 2007; Kasting, 2009; Taylor et al., 2009; Knoll et al., 2012), a pesar de haber debate en los detalles. Un elemento clave en esa historia es la emergencia de la vida, la cual ha estado interactuando con, cambiando, manteniendo y reciclando la mayoría de los materiales existentes en la atmósfera y la zona supracortical, por más del $\sim 80 \%$ de la historia de la Tierra.

Este escenario ha sido estudiado e interpretado a través de los años, con la ayuda de la tecnología disponible, no siempre correctamente y también a veces sesgado por modas 
en el consenso general (ver Hallbauer, 1975; Gray y Boucot, 1994; Windley, 2007). La apreciación de algunos fenómenos geológicos y biológicos del pasado (i.e. la formación de suelos, la sucesión ecológica, o las tasas de sedimentación) pueden ser difíciles de correlacionar temporalmente en secciones estratigráficas cuando ocurren cambios rápidos y lentos simultáneos, tales como la rápida formación de algunos volcanes, las inundaciones, los deslaves y rápidas oscilaciones climáticas, versus la lenta expansión del fondo marino, la deriva continental o la formación de montañas. A este respecto, debido a que la biología opera órdenes de magnitud más rápido que la geología, los tiempos geológicos relativamente cortos (decenas de millones de años), condensados en unos pocos centímetros o metros de estratos geológicos, pudieran representar enormes oportunidades evolutivas para los organismos, que pueden ser difíciles de reconciliar con el poco o mucho registro fósil donde estos cambios pudieran apreciarse .

Esta conceptualización de la velocidad a la que la biología opera con respecto a la geología, requiere de cuidadosos exámenes de las rocas antiguas, del avance del conocimiento científico y tecnológico en torno a éstos, pero además de una mente abierta a considerar ideas desafiantes, por ejemplo al tratar de conciliar a los fósiles con sus paleoambientes (i.e. Retallack, 2013; Xiao y Knauth, 2013 y referencias incluidas).

Con respecto a la vida terrestre, la mayoría de los microfósiles Precámbricos provienen de paleoambientes acuáticos, y aunque abundantes evidencias morfológicas (i.e. microbialitas y microfósiles), químicas (frentes de bioalteración en paleosuelos) y geoquímicas (firmas isotópicas de materia orgánica) de este registro han alcanzado una amplia aceptación y consenso, la existencia de vida propiamente terrestre en el Precámbrico no es cabalmente conocida. La percepción histórica de las plantas como el grupo dominante en la tierra, junto con los primeros descubrimientos de fósiles macroscópicos sólo en rocas Fanerozoicas y la incapacidad para interpretar correctamente biofirmas microbianas, tal vez han contribuido a la comprensión generalizada de los 'ecosistemas terrestres' exclusivamente para las plantas (i.e. Bambach, 1999). En algunos casos, incluso cuando la existencia de ecosistemas terrestres Precámbricos es reconocida, éstos se tratan dudosamente (Shear, 1991; DiMichele y Hook, 1992; Gray y Shear, 1992; Gray y Boucot, 1994; Bambach, 1999; Blackwell, 2000; Corcoran y Mueller, 2004; Nesbitt y Young, 2004; Gensel, 2008) a pesar de previas e importantes discusiones al respecto (i.e. Wright, 1985; Labandeira, 2005).

La posible malinterpretación de los paleoambientes terrestres, y su relativamente pobre conservación en el registro sedimentario, no significa que la vida terrestre fue inexistente en la Tierra primitiva. Cada vez hay más evidencia indicativa de ambientes terrestres antiguos colonizados por microbios, lo cual es coherente con la presente distribución de microbios en ambientes considerados 'estériles' en el Precámbrico (desiertos, llanuras polares, rocas alpinas, etc.). Además es notable la amplia diversidad y las capacidades metabólicas conocidas para los microbios, lo cual es también consistente con la gran diversidad y distribución de microfósiles Precámbricos conocidos (Schopf y Klein, 1992), que es un reflejo de la ubicuidad microbiana de aquel tiempo.

\section{El escenario de la vida temprana}

Los materiales más antiguos fechados hasta ahora son meteoritos y tienen 4570 millones de años (Mega annum, Ma; Bouvier y Wadhwa, 2010), y pueden servir como punto de referencia para la condensación de los primeros sólidos en el Sistema Solar y por ende la Tierra. Por el contrario, los materiales más antiguos de la Tierra (cristales de zircón) tienen $4400 \mathrm{Ma}$ (Wilde et al., 2001), dejando un hiato de $\sim 170$ Ma en la historia geológica de la Tierra. Aún así, se asume que la Luna se formó antes de los $4400 \mathrm{Ma}$ (Canup y Righter, 2000; Yu y Jacobsen, 2011) y que el núcleo de la Tierra, el manto y la litósfera ya estaban diferenciados (Nelson, 2004; Boyet y Carlson, 2005). Isótopos de oxígeno en zircones sugieren que al menos hace $\sim 4200 \mathrm{Ma}$, pero tal vez $200 \mathrm{Ma}$ antes, existieron grandes cuerpos de agua en la superficie (Mojzsis et al., 2001; Nutman, 2006; Cavosie et al., 2007, pero ver visión alternativa en Deming, 2002), mientras que cortezas graníticas (continentales) y basálticas (oceánicas) continuaban en constante crecimiento, resurgiendo y fundiéndose, interactuando con el agua en regímenes no uniformes que evolucionaron drásticamente del Hadeano al Neoarcheano (Komiya et al., 1999; Nutman et al., 2002; Myers, 2004; Rino et al., 2004; van Kranendonk, 2004 y referencias incluidas; Furnes et al., 2007a; Adam et al., 2012), cambiando de una tectónica dominada por plumas, a una dominada por placas (van Kranendonk et al., 2007). Es plausible entonces, que para el final del gran bombardeo, entre 3800 y $3900 \mathrm{Ma}$ (Gomes et al., 2005; Hartmann et al., 2000), las tierras y los océanos primitivos eran nichos abiertos listos para los microbios pioneros, para quienes las perturbaciones globales pudieron ser negligibles, dada la rápida capacidad de adaptación de la vida a sistemas cambiantes, pudiendo regenerarse y diversificarse tras los disturbios globales importantes.

Aunque la vida pudo haber existido 300 o $600 \mathrm{Ma}$ después de la acreción de la Tierra (i.e. López-García et al., 2006), el registro sedimentario más antiguo (donde los eventos bióticos son más propensos a ser conservados) no sobrepasa los $23850 \mathrm{Ma}$ (Nutman et al., 1996; Ishizuka, 2008; Nutman et al., 2010; O'Neil et al., 2011; Mloszewska et al., 2012). Y aún en este registro tan antiguo, potenciales biofirmas (carbonatos precipitados biogénicamente) pudieran estar presentes (Nutman et al., 2010), lo cual sugiere que la biósfera podría ser varios millones de años más antigua que los estromatolitos y microfósiles más antiguos conocidos ( $23500 \mathrm{Ma})$. Otras biofirmas putativas 
de más de $3500 \mathrm{Ma}$ (glóbulos grafitizados asociados a apatita; ver McKeegan et al., 2007; Papineau et al., 2010a, 2010b) son también polémicas (véase Myers, 2001; van Zuilen et al., 2002; Fedo y Whitehouse, 2002; Papineau et al., 2011) dada su dudoso tiempo de formación, que pudo ser más reciente. Firmas biológicas de particular interés son las asociadas a las llamadas Banded Iron Formations (i.e. Dauphas et al., 2004; Trendall y Blockley, 2004; Kappler et al., 2005; Konhauser et al., 2005; Koehler et al., 2010; Mloszewska et al., 2012) debido a su potencial antigüedad de $4300 \mathrm{Ma}$ (O'Neil et al., 2009).

La presencia de microfósiles, microbialitas, y biomarcadores moleculares e isotópicos en rocas de más de $3000 \mathrm{Ma}$, indican que la vida microbiana era abundante en ambientes marinos, someros y profundos del Arqueano (Lowe, 1980; Walter et al., 1980; Awramik et al., 1983; Schopf, 1983; Walter, 1983; Walsh y Lowe, 1985; Rasmussen, 2000; Westall et al., 2001; Furnes et al., 2004; Shen y Buick, 2004; Tice y Lowe, 2004; Allwood et al., 2006; Banerjee et al., 2006; Westall et al., 2006a, 2006b; Ueno et al., 2006; Schopf et al., 2007 y referencias incluidas; Shen et al., 2009; Westall, 2010; Wacey et al., 2011). Ello apoya la idea de que las zonas costeras estuarinas fueron muy productivas en aquel tiempo, y que la fotosíntesis ya estaba operando (Awramik, 1992; Rosing y Frei, 2004; Tice y Lowe, 2004; Buick, 2008; Hoashi et al., 2009; Kato et al., 2009; Kendall et al., 2010), aunque tal vez no necesariamente oxigénica (Westall et al., 2011; Li et al., 2012).

Muchos ambientes se han propuesto como probables $\mathrm{u}$ 'óptimos' para el surgimiento y la prosperidad de la vida, que van desde ventilas hidrotermales de aguas profundas y subaéreas, hasta suelos e interfases agua-sólido-gas en ambientes costeros (Baross y Hoffman, 1985; Retallack, 1986a; Holm, 1992; Battistuzzi y Hedges, 2009; Aller et al., 2010; Hazen y Sverjensky, 2010; Mulkidjanian et al., 2012). Sin embargo, los ambientes donde se encuentran la mayoría de los microfósiles Precámbricos son de origen marino somero en márgenes continentales (ver referencias en Schopf y Klein, 1992). Aunque no se sabe si esto realmente fue así o si se debe a una consecuencia del carácter incompleto o selectividad del registro geológico, los microbios de estos ambientes probablemente quedaban expuestos periódicamente a la desecación, como sucede en la mayoría de estos entornos actualmente, y probablemente desarrollaron adaptaciones para la desecación (i.e. gruesas envolturas orgánicas higroscópicas) y la alta radiación UV (i.e. viviendo intersticialmente o produciendo pigmentos protectores).

Algunos de los fósiles más antiguos provienen precisamente de ambientes costeros someros (Klein et al., 1987; Schopf y Klein, 1992; van Kranendonk et al., 2008; Westall et al., 2010; van Kranendonk, 2011; Hickman y van Kranendonk, 2012), lacustres someros (Awramik y Buchheim, 2009; Hickman y Van Kranendonk, 2012), e intermareales (Noffke et al., 2006; Noffke, 2010;
Noffke et al., 2011; Westall et al., 2011), donde signos de evaporación están presentes (Westall et al., 2011; Hickman y van Kranendonk, 2012), lo cual sugiere que las comunidades microbianas primitivas de aguas poco profundas tenían que lidiar con la desecación periódica, las fluctuaciones de salinidad y la radiación UV hace más de $3400 \mathrm{Ma}$. Es posible entonces que los organismos primitivos desarrollaran adaptaciones para vivir en ausencia de, y lejos del agua. Lo mismo aplicaría para comunidades de ambientes lacustres y fluviales expuestas a desecación.

La desecación favorece a su vez la dispersión por viento, lo cual pudo ser un mecanismo clave para la colonización de los continentes. A través de la dispersión por viento, las comunidades colonizadoras tenderían a estar más en la superficie que bajo tierra, a pesar de posibles migraciones a los acuíferos de toda índole. Es probable entonces que los ambientes expuestos a la desecación (especialmente estuarios y zonas intermareales) fueran escenarios cruciales para una transición biológica entre loa ambientes acuáticos y los subaéreos.

Aparentemente no sólo los procariontes eran abundantes en ambientes someros Precámbricos. Los fósiles tipo eucarionte más antiguos (acritarcos, Buick, 2010), que quizás requerían oxígeno para maximizar sus capacidades energéticas y metabólicas, tienen $\sim 3200$ Ma de edad y también estuvieron presentes en ambientes estuarinos (Javaux et al., 2010). A pesar de desconocer su verdadera identidad, la presencia de microfósiles de gran tamaño ( $>$ $150 \mu \mathrm{m}$ de diámetro celular) indica que la vida se diversificó y alcanzó una presencia global relativamente rápido (Kandler, 1994; Altermann y Schopf, 1995; Ueno et al., 2006; Blank, 2009; David y Alm, 2011), y ocupó una amplia variedad de nichos ecológicos hacia el Paleoarcheano, incluso en lugares gravemente perturbados por impactos de asteroides (ver Walsh, 1992 y referencias incluidas). Una mayor diversidad, ubicuidad, y abundancia biológica aparece más adelante en el tiempo, en el Proterozoico tardío (i.e. Schopf, 1992a; Schopf y Klein, 1992), cuyo registro geológico está mejor conservado y es más abundante que el del Arqueano.

\section{El registro fósil de la vida terrestre}

Los restos más antiguos de corteza continental derivan de zircones (Nutman, 2006) y afloramientos regionales (Buick et al., 1995; Iizuka et al., 2006; Stern y Scholl, 2010; Adam et al., 2012) de $\geq 3500 \mathrm{Ma}$ de edad. La evidencia complementaria de exposición subaérea consiste en extensos suelos desarrollados sobre algunas de estas antiguas superficies (Buick et al., 1995; Hoffman, 1995; Johnson et al., 2009, 2010). El paulatino crecimiento de los continentes (i.e. Santosh, 2010) y la resultante expansión de zonas subaéreas se refleja en el amplio espectro de paleosuelos Proterozoicos (ver métodos de estudio y ejemplos en Jackson, 1967; Gay y Grandstaff, 
1980; Holland, 1984; Aspler y Donaldson, 1986; Grandstaff et al., 1986; Kimberley y Grandstaff, 1986; Reimer, 1986; Retallack, 1986b; Farrow y Mossman, 1988; Zbinden et al., 1988; Palmer et al., 1989; Holland, 1992; Gall, 1994; Macfarlane et al., 1994; Martini, 1994; Retallack y Mindszenty, 1994; Driese et al., 1995; Banerjee, 1996; Ohmoto, 1996; Prasad y Roscoe, 1996; Gutzmer y Beukes, 1998; Thiry y Simon-Coincon, 1999; Rye y Holland, 2000; Watanabe et al., 2000; Retallack, 2001 y referencias incluidas; Yang y Holland, 2003; Driese y Gordon-Medaris, 2008; Pandit et al., 2008; Bandopadhyay et al., 2010). Este registro de paleosuelos contiene información indirecta sobre las condiciones del medio terrestre primitivo y conserva firmas geoquímicas que pudieran demostrar una cubierta biológica asociada a éste.

Actualmente, la evidencia más antigua y directa de vida terrestre proviene de paleosuelos y secuencias aluviales de 2900-2700 Ma de edad en Sudáfrica (ver determinación de la edad de los depósitos de Witwatersrand en Kositcin y Krapez, 2004; Zhao et al., 2006), ricos en materia orgánica y microfósiles (Hallbauer y van Warmelo, 1974; Mossman et al., 2008), así como pseudomicrofósiles y paleoturberas de 2700 Ma en Australia (Rye y Holland, 2000) y firmas isotópicas de carbono orgánico de paleosuelos en Sudáfrica (Watanabe et al., 2000). Coincidentemente, este registro co-ocurre con a) cambios drásticos en la configuración de la corteza y el, tal vez abrupto, emplazamiento de grandes masas continentales en el Arqueano tardío (Condie, 2004; Eriksson y Martins-Neto, 2004; van Kranendonk, 2004 y referencias incluidas; Hazen et al., 2012), b) una probable oxigenación de la atmósfera (Kendall et al., 2010), y c) estimaciones de la colonización terrestre por microbios según sus relaciones filogenéticas (Battistuzzi et al., 2004). Aunque los microbios podrían haber colonizado los ambientes terrestres antes de este tiempo, pareciera que del Meso- al Neoarcheano ocurren cambios importantes en la distribución y diversidad de comunidades microbianas terrestres. De éstos, tal vez el más relevante supone la historia del crecimiento de los supercontinentes (Santosh, 2010) y la resultante aparición de nuevos hábitats colonizables.

A medida que el registro geológico se hace más reciente, la cantidad de paleosuelos ricos en materia orgánica y posiblemente biológicamente intemperizados (Ohmoto, 1996; Beukes et al., 2002; Driese y Gordon-Medaris, 2008), las estructuras biosedimentarias terrestres (Hupe, 1952; Lannerbro, 1954; Voigt, 1972; Eriksson et al., 2000; Prave, 2002) y los microfósiles (Cloud y Germs, 1971; McConnell, 1974; Horodyski y Knauth 1994; Strother et al., 2011) aumentan drásticamente en abundancia, distribución y diversidad en el Proterozoico. Del mismo modo, microfósiles marinos muestran crecientes desarrollos biológicos y adaptaciones durante este periodo (Knoll et al., 2006), sobre todo hacia la transición NeoproterozoicoFanerozoico (Zhuravlev y Riding, 2001; Xiao y Kaufman, 2006; Gaucher et al., 2010), cuando por primera vez aparecen los animales macroscópicos. Esta cronología sugiere un desarrollo rápido y global de la vida en la Tierra, con formas de vida adaptadas a vivir en ambientes terrestres más de $2000 \mathrm{Ma}$ antes del primer registro fósil de plantas terrestres (Heckman et al., 2001; Gensel, 2008). Eventos importantes en esta cronología se muestran en la Figura 1.

\section{Funcionamiento de los ecosistemas terrestres primitivos}

Conceptualizar el funcionamiento de la biósfera terrestre antigua requiere necesariamente una comprensión general del funcionamiento y distribución de las comunidades microbianas modernas, análogas, para conocer su dinámica, diversidad, fisiología, e impacto ambiental, y así poder extrapolar interpretaciones con cierto grado de certeza. De igual importancia resulta caracterizar cualquier potencial biofirma, para luego reconocerlas en las rocas.

Las comunidades microbianas terrestres se encuentran hoy en todo el mundo y abarcan una gran variedad de condiciones ambientales y estacionales. Los ambientes continentales podrían ser divididos en superficiales (roca, regolito) y subsuperficiales (cuevas, acuíferos, suelo profundo). Comparativamente entre ambos, no está claro cuál es más productivo en términos de producción de biomasa (Pace, 1997), ni qué metabolismos, y en qué medida, han dominado tales sistemas durante escalas de tiempo geológico (Sleep y Bird, 2007).

Comprender la biología y distribución de los microbios modernos, que son omnipresentes en la biósfera actual (Fig. 2), resulta esencial para comprender su pasado y su impacto en los ecosistemas terrestres a través del tiempo. Estimaciones de la diversidad genética y distribución de biomasa en ambientes drásticamente distintos (i.e. GarciaPichel et al., 2003; Lozupone y Knight, 2007; Nemergut et al., 2011) muestran la amplia gama de estrategias que los organismos terrestres, en particular los productores primarios, han desarrollado para vivir en la tierra. La fotosíntesis oxigénica domina los sistemas terrestres y parece ser una adaptación muy importante evolutivamente, pues su fuente de energía (luz), el poder reductor (agua), y la fuente de carbono $\left(\mathrm{CO}_{2}\right)$ se consiguen fácilmente en estos entornos. En comparación, otros productores primarios (por ejemplo, quimiolitótrofos) están restringidos a ambientes acuáticos debido a que requieren agentes reductores solubles (i.e. $\mathrm{H}_{2}, \mathrm{Fe}^{2+}, \mathrm{H}_{2} \mathrm{~S}, \mathrm{HS}^{-}$) para mantener su metabolismo (White, 2000). Además de estar restringidos a ambientes acuáticos, también son menos eficientes que los fotoautótrofos oxigénicos (DesMarais, 2000; Madigan et al., 2003; Konhauser, 2007).

Las cianobacterias fueron los únicos organismos que desarrollaron pigmentos y enzimas para acoplar la energía fotónica a la oxidación del $\mathrm{H}_{2} \mathrm{O}$ para la obtención de electrones y subsecuente almacenamiento de energía y producción de biomasa (i.e. White, 2000). Este proceso les ha permitido vivir en ambientes subaéreos, incluso donde 


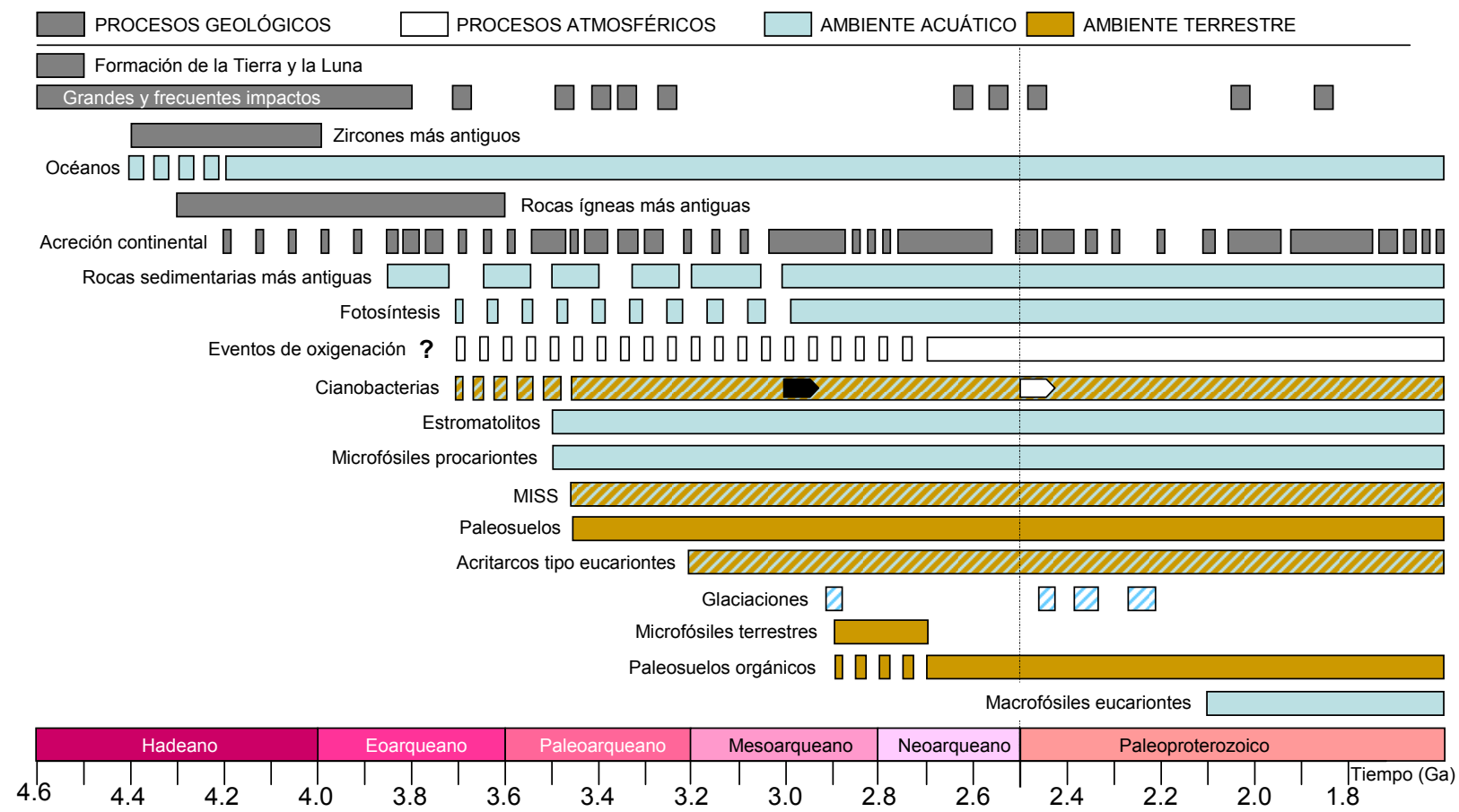

Figura 1. Cronología sugerida de eventos geológicos, atmosféricos y biológicos durante los primeros 3000 Ma desde la acreción de la Tierra. Los eventos geológicos fueron compilados a partir de las referencias incluidas en Canup y Righter (2000), Eriksson et al., (2004), y van Kranendonk et al., (2007). Historia de los impactos de asteroides por Glikson (2007). Emplazamiento de los océanos por Nutman (2006). La línea vertical a los 2500 Ma marca un brusco incremento en la oxigenación de la atmósfera (ver Kendall et al., 2010), aunque el $\mathrm{O}_{2}$ pudo acumularse antes (Ohmoto, 2004). Los eventos de oxigenación se consideran ocurridos en pulsos de magnitud y duración desconocida, y se correlacionan con el origen de la fotosíntesis oxigénica por las cianobacterias, basado en estimados por Lazcano y Miller (1994), y teniendo en cuenta la aparición de la vida hace 3800 Ma. En la línea de las cianobacterias, dos orígenes alternativos se indican con una marca negra (Schirrmeister et al., 2013) y una blanca (Kirschvink y Kopp, 2008). Las MISS (Microbially Induced Sedimentary Structures, ver Noffke et al., 2001), las cianobacterias, y los acritarcos tipo eucarionte unicelulares están representados en ambos dominios, acuático y terrestre. Las glaciaciones son de Hoffman y Schrag (2002), y se muestran en colores combinados para representar procesos atmosféricos (climáticos) e hidrológicos (formación de hielo). Los principales pasos evolutivos biológicos y los datos relacionados con paleosuelos son de Hallbauer y van Warmelo (1974), Holland (1984), Schopf (1983), Schopf y Klein (1992), Han y Runnegar (1992), Golubic y Seong-Joo (1999), Rye y Holland (2000), Retallack (2001), Westall et al., (2006a, b), Johnson et al., (2009, 2010), El Albani et al., (2010), Javaux et al., (2010).

el agua es un factor limitante, como en los desiertos (i.e. Potts y Friedmann, 1981). La fotosíntesis oxigénica también contribuyó a la oxidación de la atmósfera (secuestrando $\mathrm{CO}_{2}$ y produciendo $\mathrm{O}_{2}$ ), un fenómeno global y continuo, con profundas repercusiones geoquímicas, atmosféricas, hidrológicas y biológicas (i.e. Rosing et al., 2006; Och y Shield-Zhou, 2012; Pufahl y Hiatt, 2012). Muchos procariontes, incluidas las cianobacterias, también pueden fijar el nitrógeno gaseoso $\left(\mathrm{N}_{2}\right)$, lo cual resulta ventajoso para prescindir de especies disueltas como $\mathrm{NH}_{4}$ y $\mathrm{NO}_{3}$ (Glass et al., 2009) o aminoácidos preexistentes. La aparición de acinetos fósiles de cianobacterias (para la fijación de $\mathrm{N}_{2}$ ) en el Paleoproterozoico (Tomitani et al., 2006) sugiere que esta adaptación fue temprana. Otros nutrientes limitantes, como el P, pueden ser suministrados via polvo atmosférico (Kennedy et al., 1998; Reynolds et al., 2001; McTainsh y Strong, 2007), que puede ser un proceso alternativo para la reposición de la pérdida de nutrientes por lixiviación, escorrentía y filtración en estos entornos (Beraldi-Campesi et al., 2009). El S también puede ser ubicuo en minerales (sulfuros y sulfatos), aerosoles y gases (i.e. $\mathrm{H}_{2} \mathrm{~S}$ y $\mathrm{SO}_{2}$ ), seguramente presentes en la atmósfera primitiva (Holland,
1984). Por lo tanto, los requerimientos nutricionales para los productores primarios (en este caso fotosintéticos oxigénicos) no parecen haber sido un factor limitante para la colonización de los continentes. Esta idea también se ha discutido a la luz de las características fisiológicas y genéticas de microbios terrestres (Battistuzzi et al., 2004; Battistuzzi y Hedges, 2009), que plantean el origen de las cianobacterias a partir de organismos terrestres, no acuáticos. Sin embargo, es posible que formas de vida quimiotrófica más primitivas (Shen y Buick, 2004; Sleep y Bird, 2007) hayan sido dominantes en cuerpos de agua continentales antes de la invención de la fotooxidación del agua.

En particular, para la biota terrestre temprana, un conjunto mínimo de adaptaciones para vivir subaéreamente debe haber incluido la protección contra los efectos de la radiación y la desecación. Adaptaciones como vainas de exopolisacáridos (EPS) gruesas con capacidad higroscópica contra la desecación, mecanismos de reparación de ADN y eficientes mecanismos de restauración metabólica cuando hay disponibilidad de agua, y la producción de pigmentos de blindaje contra luz UV, son ciertamente exitosas estrategias 


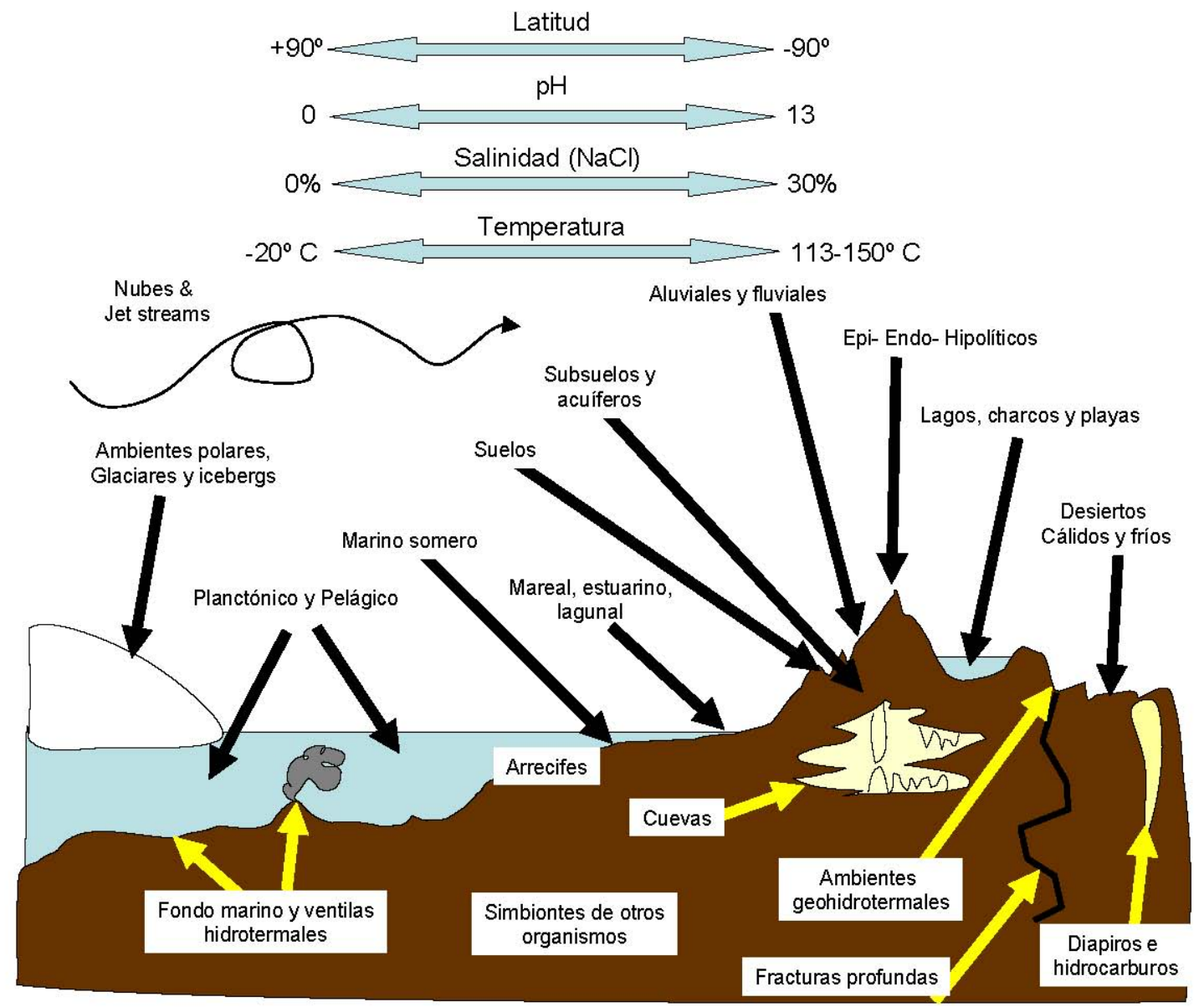

Figura 2. Variedad de ambientes conocidos donde habitan microbios. Los rangos de las fluctuaciones ambientales (latitud, pH, salinidad y temperatura) donde los microbios pueden ser encontrados también se muestran (ver Madigan y Marrs, 1997; Madigan et al., 2003; Konhauser, 2007). Interacciones directas e indirectas entre las diferentes comunidades microbianas, así como asociaciones simbióticas, coevolución e intercambio genético horizontal (i.e. Davison, 1999; Gogarten et al., 2007) se asumen como procesos que impulsaron la evolución de procesos ecológicos y adaptaciones a nuevos hábitats.

mostradas por las cianobacterias terrestres (i.e. Shephard, 1987; García -Pichel, 1998; Yasui y McCready, 1998; Potts, 1999; Sinha y Häder, 2002; Singh et al., 2010). Algunos de estos refinados mecanismos de adaptación a la vida subaérea incluyen la producción de pigmentos que, una vez colocados fuera de la célula dentro de las vainas extracelulares, proteger pasivamente contra la radiación UV, incluso cuando las células están inactivas o deshidratadas (GarcíaPichel y Castenholz, 1991; Gao y García-Pichel, 2011). Algunas de estas estrategias probablemente evolucionaron temprano y son parcialmente mostradas por microfósiles (por ejemplo, vainas gruesas y 'pigmentadas'), que a veces están asociados con sedimentos evaporíticos, coincidiendo con la exposición subaérea (Schopf, 1968; Hofmann, 1976; Golubic y Campbell, 1979; Awramik et al., 1983).

Dada su potencial antigüedad y dominancia espacial, las cianobacterias resultan candidatos ideales para la colonización de los continentes Precámbricos. Las comunidades de cianobacterias modernas pueden encontrarse en cualquier medio terrestre $(\sim 30 \%$ de la superficie del planeta), sobre rocas (i.e. en ambientes endolíticos; Friedmann, 1980; Sun y Friedmann, 1999; Büdel et al., 2004.) y en suelos (Belnap y Lange, 2001). Las cubiertas criptogámicas (Cryptogamic Ground Covers o CGC; Elbert et al., 2012) han demostrado ser sistemas complejos y dinámicos que contienen varios grupos funcionales distintos de procariontes y eucariontes, desde los productores primarios hasta los descomponedores de materiales específicos y los herbívoros (Fritsch, 1922; Fletcher y Martin, 1948; Campbell, 1979; Bamforth, 1984, 2004; Garcia-Pichel et al., 2001; Nagy et al., 2005; Tirkey y Adhikary, 2005; Chanal et al., 2006; Reddy y Garcia-Pichel, 2006; Bates y Garcia-Pichel, 2009; Neher et al., 2009; Meadow y Zabinski, 2012). Esta diversidad es variable según las condiciones ambientales locales, pero todos tienen en común la presencia de cianobacterias, con pocas excepciones (i.e. Hoppert et al., 2004; Smith et al., 2004).

Aunque existen análogos fósiles de CGC (Simpson et al., 2010; Beraldi-Campesi et al., 2011; Retallack, 2009, 2011; Sheldon, 2012), no se sabe cuál fue su composición microbiana. Comparativamente, cualquier costra biológica del suelo es menos resistente que los tapetes microbianos 
acuáticos, simplemente porque la disponibilidad limitada de agua impide el crecimiento rápido en la primera. Ello supone una menor probabilidad de fosilización en ambientes áridos que en ambientes húmedos. Sin embargo, comparaciones morfológicas entre estructuras biosedimentarias (también llamadas MISS -Microbially Induced Sedimentary Structures-; Noffke et al., 2001) producidas en CGC modernas y fósiles son notables (Schieber et al., 2007; Noffke, 2010). Esta semejanza morfológica entre MISS fósiles y recientes sugieren que las cianobacterias son de hecho un grupo muy antiguo (Golubic y Seong-Joo, 1999) y que por lo menos algunas características morfológicas se han mantenido en el tiempo (Golubic y Hofmann, 1976; Golubic y Campbell, 1979; Schopf, 1992b; Noffke, 2010). Dado el antiguo linaje de las cianobacterias y dadas sus extraordinarias adaptaciones para colonizar sedimentos inestables (Booth, 1941; Campbell et al., 1989; Mazor et al., 1996; Belnap y Gillette, 1998; Malam-Issa et al., 2001; Hu et al., 2002; Garcia-Pichel y Wojciechowski, 2009), en sitios donde el agua es escasa y la radiación UV considerable (Fleming y Castenholz, 2007; Giordanino et al., 2011), suponen candidatos naturales para la colonización de las superficies terrestres antiguas (Campbell, 1979). Parece lógico entonces que este tipo de comunidades hayan influido en la formación de estructuras y texturas biosedimentarias representadas en rocas antiguas terrestres (i.e. Prave, 2002; Schieber et al., 2007). La antigüedad de las cianobacterias también ha sido estimada, con distancias genómicas y relojes moleculares, en 2000 Ma (Battistuzzi y Hedges, 2009; Schirrmeister et al., 2013), que más o menos coincide con la edad de los microfósiles terrestres más antiguos (Mossman et al., 2008). Estos estimados, sin embargo, pueden variar dependiendo de los puntos de calibración utilizados para la construcción de filogenias y del intercambio genético que hayan tenido el linaje en el tiempo.

A pesar de que muchos detalles de la evolución de las cianobacterias no están resueltos, su capacidad de sintetizar clorofila a para absorber energías fotónicas más altas que otras bacterioclorofilas (Xiong et al., 2000), puede deberse a una presión selectiva para usar tales longitudes de onda que alcanzaban la superficie Precámbrica donde las cianobacterias tenían que vivir, contrario a las bacterias fototróficas púrpuras o verdes que utilizan menores longitudes de onda en sus hábitats sumergidos y protegidos. Así, desde una perspectiva multi-angular, las cianobacterias parecen ser organismos bien adaptados para la colonización de las primeras superficies terrestres.

Como se mencionó anteriormente, la mayoría de las CGC tienen en común la presencia de cianobacterias filamentosas. Una característica de estos morfotipos es que pueden deslizarse a través de los espacios intersticiales utilizando vainas huecas de mucílago como senderos, para protegerse intersticialmente contra la radiación, y para buscar su régimen óptimo de luz y de agua (Garcia-Pichel y Pringault, 2001). Estas vainas representan importantes elementos estructurales en la formación de 'costras biológicas' en ambientes siliciclásticos. La naturaleza filamentosa de los organismos también ofrece una mayor superficie celular y fuerza tensil para fijar y unir partículas disgregadas en el suelo (Garcia-Pichel y Wojciechowski, 2009). Polisacáridos secretados extracelularmente proporcionan fuerza adicional de adhesión entre partículas, lo que resulta en la formación de un microambiente estable (costra), que puede mantenerse en el tiempo y resistir a las fuerzas erosivas. En este sentido, la característica intrínseca de los microorganismos filamentosos para formar capas cohesivas en las superficies sedimentarias disminuye sustancialmente la erosión del viento y el agua en zonas áridas y semiáridas del mundo (Belnap y Gillette, 1998; Belnap y Lange, 2001). Aunque algunas fuerzas erosivas pueden superar la resistencia de las $\mathrm{CGC}$ en sistemas de alta energía (i.e. Corcoran y Mueller, 2004), esta propiedad de resistencia ha sido invocado para explicar la estabilidad y el espesor de secuencias sedimentarias siliciclásticas Precámbricas (Dott, 2003) y propiedades de 'deformación suave' (soft deformation) en tapetes microbianos fósiles (ver referencias en Schieber et al., 2007). Esta es una propiedad importante de los microbios modernos en el funcionamiento de los ecosistemas siliciclásticos, y junto con la presencia de suelos Proterozoico maduros y ricos en materia orgánica y microfósiles (ver referencias anteriores), sugieren la presencia de abundantes CGC en el Precámbrico, similares a las que cubren las zonas polares y áridas del mundo actual. La posible incorporación de nuevos miembros a estas comunidades a través del tiempo (sobre todo algas y hongos) podrían explicar el incremento en las tasas de meteorización de los continentes (Kennedy et al., 2006) y los cambios bruscos en el equilibrio global del $\mathrm{C}$ en el Neoproterozoico (Knauth y Kennedy, 2009).

\section{Otros componentes microbianos terrestres}

A juzgar por la rápida diversificación y distribución de la biota microbiana temprana, así como por sucesiones microbianas en ambientes modernos, incluidos aquellos con baja producción primaria (i.e. Sigler et al., 2002; Schmidt et al., 2008; Fierer et al., 2010), se espera que organismos heterótrofos también formaran parte de las comunidades terrestres primitivas, ya que parecen ser un complemento inevitable en estos consorcios. Bajo esta perspectiva, los ecosistemas microbianos primitivos estarían compuestos por productores primarios autótrofos, pero también por una miríada de otros microbios que encuentran su nicho en microambientes pre-existentes. Por ejemplo, algunas actinobacterias que habitan suelos, no sólo degradan grandes cantidades de exudados orgánicos de las cianobacterias, lo cual influye en el ciclo del $\mathrm{C}$, sino que también parecen ser componentes estructurales dentro de las CGC (i.e. Reddy y Garcia-Pichel, 2006). Lo mismo se aplica para otros taxones (i.e. Bacteroidetes y Proteobacteria) que secretan grandes cantidades de mucopolisacáridos, que 
a su vez ayudan a adherir las partículas del suelo (que forma la 'costra' biológica) y tener un papel crítico en la conductividad hidráulica de la superficie del substrato (Rossi et al., 2012). Uno de los componentes más importantes de eucariontes de CGC modernos son los hongos, que deben haber jugado un papel clave en la colonización y la erosión de las rocas desnudas en el pasado (i.e. líquenes con cianobacterias o algas como simbiontes), así como en la simbiosis con plantas vasculares (Smith y Read 2008), que cambiaron radicalmente los ecosistemas terrestres más 'modernos' (Blackwell, 2000, Heckman et al., 2001; Gadd, 2006; Taylor et al., 2009). Así, los consorcios microbianos resultan importantes en todos los aspectos relacionados con el desarrollo de ecosistemas terrestres, pues coevolucionan simultáneamente en microhábitats que influencian al macrohábitat.

Aunque la temporalidad en la ocurrencia de estos organismos en el tiempo es desconocida y su registro fósil limitado, la existencia de cubiertas microbianas en los continentes antiguos supone efectos de alto impacto en escalas de tiempo geológico. Por ejemplo, es conocido que los microbios terrestres pueden conducir importantes transformaciones químicas en suelos (Keller y Wood, 1993; Schwartzman y Volk, 1989; Chenu y Stotzky, 2002; Ehrlich, 2002; Chorover et al., 2007) y hábitats endolíticos (Konhauser et al., 1994; Sun y Friedmann, 1999; Büdel et al., 2004; Omelon et al., 2006), al afectar la reactividad de las superficies minerales con metabolitos secretados extracelularmente (Geesey y Jang, 1990; Welch et al., 1999 ), cambiando el potencial redox y $\mathrm{pH}$ de su microentorno (Bennett et al., 2001), o secretando ligandos/quelantes de metales y otros complejos orgánicos que reaccionan con los solutos y minerales del suelo (Keller y Wood, 1993; Schwartzman y Volk, 1989; Barker et al., 1998; Welch et al., 1999; Bennett et al., 2001). Estos mecanismos parecen jugar un papel fundamental en la biogeoquímica del suelo (erosión, formación de arcillas, biodisponibilidad y concentración de nutrientes, formación o transformación de minerales, etc.), y sus efectos pudieran ser también manifestarse como biofirmas geoquímicas para rastrear microbios en las rocas (Beraldi-Campesi et al., 2009). Además, la formación y maduración del suelo se relaciona estrechamente con la biología (Keller y Wood, 1993; Schwartzman y Volk, 1998, Brady y Weil, 2008), a diferencia de los procesos abióticos que forman regolito, lo cual supone un paso fundamental previo a la colonización terrestre por plantas y animales. Todas estas características mostradas por CGC modernos podrían esperarse de comunidades análogas antiguas, aunque con variaciones en la incidencia y magnitud de sus impactos geobiológicos, dictados en parte por los factores limitantes del medio.

\section{El polvo}

El mecanismo de formación de polvo, su transporte y deposición, refleja un aspecto importante para el funcionamiento de los ecosistemas terrestres, y también para los marinos, porque el polvo sólo se forma en los ambientes subaéreos, y porque los microbios (junto con la adhesión por agua y la neo-cementación de partículas con sales y arcillas) pueden estabilizar las partículas finas de polvo a través de su captura y cementación o trapping and binding (i.e. Dong et al., 1987; Liu et al., 1994; Williams et al., 1995; Belnap y Gillette, 1998; Hu et al., 2002). Por lo tanto, la producción de polvo puede ser regulada potencialmente por microbios en función de su grado de desarrollo. Cuanto más desarrolladas son las CGC, menor es la producción de polvo.

El polvo es un importante portador de nutrientes y su retención en el suelo puede influir en el balance nutricional local, o de ecosistemas lejanos, como sucede actualmente en medios marinos tras la deposición de grandes cargas de polvo (Jickells et al., 2005). La capacidad de los microbios para capturar y unir partículas ha sido demostrada para numerosos entornos subacuáticos y subaéreos (Gunatilaka, 1975; Zhang, 1992; Takeuchi et al., 2001; Altermann, 2008; Gradzinski et al., 2010; Williams et al., 2012). Que los microbios fueran responsables de gran parte de la captura global de polvo, retención y lixiviación en los continentes primitivos, tendría profundas implicaciones para el funcionamiento biogeoquímico y evolución de los ecosistemas globales a través del tiempo, así como para importantes procesos climáticos (i.e. variaciones del albedo atmosférico; Harrison et al., 2001; Jickells et al., 2005; Lau y Kim, 2006).

Por último, el polvo es también un portador de microbios y virus (Abed et al., 2011; Al-Bader et al., 2012), lo que implica un medio de dispersión biológica que debe haber funcionando de forma continua y a larga distancia en el pasado, amplificando así la biogeografía potencial de entidades biológicas sobre océanos y continentes. Sin embargo, la tasa de supervivencia y la prosperidad de las comunidades acarreadas por viento en ambientes acuáticos, o superficies continentales estériles o colonizadas, no se conoce, pero es posible que ese mecanismo fuera vital para la dispersión biológica terrestre y el incremento de su complejidad ecológica a través del intercambio genético (i.e. Gogarten et al., 2007) entre especies de distintas zonas geográficas.

\section{Los ambientes subterráneos}

Los ambientes subterráneos (venas/conductos geotérmicos, acuíferos, suelos y rocas profundas, cuevas de todo tipo) también deben ser considerados potenciales hábitats para la vida terrestre primitiva, donde es abundante en la actualidad (i.e. Ghiorse y Wilson, 1988; Barton y Northup, 2007; Engel, 2010). El registro Precámbrico de cuevas (i.e. entornos kársticos) o acuíferos subterráneos (detectados a través de nódulos y concreciones en las rocas) es mucho menos conocido que los típicos ambientes 
marinos o lacustres (ver ejemplos de ambientes kársticos y subterráneos en Glover y Kah, 2006; Skotnicky y Knauth, 2007; Rasmussen et al., 2009). Sin embargo, estos ambientes deben haber existido a lo largo de la historia de la Tierra, y por lo tanto, biotas terrestres podrían haberse adaptado a vivir en esas condiciones en el Precámbrico (Rasmussen et al., 2009).

En contraste con los ambientes subaéreos dominados por comunidades fotosintéticas, los microbios subterráneos requieren un metabolismo quimiosintético para la productividad primaria, tal vez confinado a la oxidación de compuestos con azufre y hierro (principales fuentes de energía de tales ambientes) para sustentar su crecimiento y continuidad (Sarbu et al., 1996; Chen et al., 2009; Porter et al., 2009). Debido a que estas vías metabólicas son menos energéticas que la fotosíntesis (i.e. White, 2000), es probable que la vida bajo tierra se desarrollara más lentamente y fuera menos dinámica en términos de diversidad, complejidad de interacciones, y dispersión geográfica. Sin embargo, los primeros habitantes subterráneos pudieron haber afectado, a largo plazo, el subsuelo (formación de cuevas, alteración de hidrocarburos, producción de gases como $\mathrm{CH}_{4}, \mathrm{CO}_{2} \mathrm{y} \mathrm{H}_{2} \mathrm{~S}$ ) y contribuyó a la neoformación y disolución de minerales, así como con la generación de subproductos gaseosos que podrían ser importantes para los procesos geoquímicos en la superficie, y en última instancia para comunidades distantes y el reciclaje biogeoquímico global. Por otra parte, este tipo de entornos podría haber estado mejor protegido de crisis ambientales drásticas y globales que los ambientes subaéreos, y así funcionado como reservorios biológicos con especies que posteriormente podrían explotar ambientes habitables superficiales.

\section{Nota sobre biofirmas}

Los vestigios de vida que se encuentran en las rocas pueden formarse de varias maneras y pueden ser reconocidos siempre y cuando tales rocas se hayan conservado y sean accesibles. Aunque este registro 'fósil' se reduce entre más antiguas son las rocas, diferentes tipos de biofirmas se han podido encontrar en rocas sedimentarias (Schopf, 1983; Schopf y Klein, 1992; Schieber et al., 2007; Noffke, 2010), ígneas (Banerjee et al., 2006; Furnes et al., 2004, 2007b; Fliegel et al., 2010) y metamórficas (Franz et al., 1991; Hanel et al., 1999; Squire et al., 2006; Bernard et al., 2007; Schiffbauer et al., 2007, 2012; Schiffbauer y Xiao, 2009; Zang, 2007), de todas las edades.

La preservación de biofirmas ocurre en cualquier ambiente, pero es favorecida en ambientes subacuáticos donde la oxidación de la materia orgánica ocurre más lentamente que en ambientes subaéreos. Asimismo habrá mejor preservación si los materiales biológicos son enterrados rápidamente, si el sedimento es de grano fino, y si las condiciones microambientales son en general reductoras y anóxicas. Todos estos factores promueven la rápida permineralización y sustitución de los materiales biológicos por minerales (Farmer, 1999; Zonneveld et al., 2010; Allison y Bottjer 2011, Lalonde et al., 2012), que puede preservar la morfología y restos orgánicos, aunque esto no significa que la preservación siempre ocurre (Zonneveld et al., 2010 y referencias incluidas). De no estar protegida, la materia orgánica tiende a degradarse por fotoquímica (si se expone a la luz), ruptura de enlaces químicos, reciclaje biológico, maceración mecánica y disolución. Aún si se conservan fósiles, muchas veces la falta de morfologías diagnósticas para la mayoría de las bacterias y la posible existencia de morfologías abióticas similares a microbios (García-Ruiz et al., 2002, 2003) hacen de su identificación y determinación taxonómica un verdadero desafío. Sin embargo, su presencia en un adecuado contexto geológico y su asociación con estructuras biosedimentaria pueden utilizarse como criterios para establecer su biogenicidad. Biomarcadores moleculares en hidrocarburos que pueden ser correlacionados con organismos existentes (ej. Summons et al., 1999) también requieren de cuidadosas confirmaciones de singenicidad para una correcta interpretación (Rasmussen et al., 2008; Brocks, 2011).

Si hay factores limitantes en juego (ej. falta de agua, nutrientes, etc.), las comunidades microbianas tendrán dificultad para desarrollar la biomasa suficiente y ejercer efectos biogeoquímicos tangibles como para producir una biofirma (ya sea química, geoquímica, mineralógica, o morfológica). El agua, por ejemplo, que es un requisito básico para la supervivencia y la reproducción de las células, tiende a ser un factor limitante en ambientes terrestres, comparado con un cuerpo de agua permanente. Si el crecimiento microbiano es limitado, por tanto, la cantidad de células y biomasa que pueden ser conservados en el registro fósil también disminuye. Así, los organismos con acceso a recursos ilimitados serían capaces de crecer comunidades más grandes y tener más posibilidades de fosilización. Ello contrasta, por ejemplo, con microbios terrestres que dependen mayoritariamente del rocío o la lluvia para su supervivencia y mantenimiento. Por ejemplo, el espesor y cohesión de un tapete microbiano marino intermareal (ver Bauld, 1981; Bauld et al., 1992) son mayores que en una costra biológica madura (Belnap y Lange, 2001), por lo que esta última será menos propensa a la fosilización que su contraparte marina. Sin embargo, bajo climas y dinámicas sedimentarias favorables, incluso las frágiles costras biológicas pueden conservarse (ej. Prave, 2002; Simpson et al., 2013). Hacen falta más estudios sobre biofirmas producidas por microbios terrestres para poder compararlas contra el registro geológico todavía por explorar.

\section{Conclusiones}

A medida que la Tierra fue evolucionando, la desgasificación y gradual acumulación de agua líquida en su superficie fue diferenciando entornos acuáticos y 
no acuáticos. Es posible que la vida haya evolucionado en ambientes terrestres, paralelamente a la vida acuática (ver Retallack, 1986a y referencias incluidas). Independientemente de cómo haya sucedido esto, la vida terrestre debió necesitar adaptaciones especiales, tales como la capacidad de adquirir nutrientes y fuentes de energía fuera del ámbito acuático, mecanismos moleculares de reparación celular, y protección contra la radiación y la desecación. Estas adaptaciones se encuentran en un gran número de microorganismos, pero notablemente en las cianobacterias, un grupo con un linaje biológico muy antiguo y que incluye a los productores primarios más visibles y exitosos de la Tierra (i.e. Whitton y Potts, 2000; Herrero y Flores, 2008).

La evidencia directa e indirecta que apunta a ambientes terrestres habitados desde el Paleoarcheano (Johnson et al., 2009,2010 ) y las épocas siguientes (Stüeken et al., 2012), junto con la evidencia sustantiva de terrestrialización a partir del Neoarcheano (Hallbauer and Warmelo, 1974; McConnell, 1974; Horodyski y Knauth, 1994; Gutzmer y Beukes, 1998; Rye y Holland, 2000; Watanabe et al., 2000; Prave, 2002; Rasmussen et al., 2009), implica fuertemente la presencia de ecosistemas terrestres funcionales en el Precámbrico temprano. Las implicaciones de tal colonización no han sido completamente resueltas, pero los efectos de la vida microbiana terrestre en los procesos que afectan a la atmósfera, la litosfera y la hidrósfera, son muy diversos y actúan en diferentes escalas y niveles. Dos de estos efectos son la oxigenación continua de la atmósfera (con consecuencias para la estratificación de los océanos, la formación y el mantenimiento de la capa de ozono, la precipitación de óxidos y otros minerales, etc.) y la erosión de los continentes, que directa e indirectamente afectan a los ecosistemas marinos (i.e. Holland, 1984; Catling et al., 2001; Stüeken et al., 2012).

Existe una enorme importancia en el establecimiento de la vida terrestre para la evolución de la biósfera en el tiempo, en contraste con la biota marina que afecta indirectamente a los ecosistemas terrestres a través de procesos atmosféricos (incluyendo la composición del gas y el clima), porque los subproductos gaseosos producidos desde los continentes serían liberados directamente a la atmósfera y no disueltos primero en el océano. El paso directo del oxígeno a la atmósfera, sin residir en el océano, implica un funcionamiento distinto de la atmósfera al propuesto comúnmente en la literatura (i.e. Kasting, 2009), dado que, una vez en la atmósfera, el oxígeno podría reaccionar con moléculas reducidas antes de comenzar a acumularse en el océano y producir firmas geoquímicas en las rocas (i.e. Lyons y Gill, 2010). Por lo tanto, la vida terrestre podría haber sido fundamental para la oxigenación de la atmósfera temprana, que más tarde afectó a los océanos también. Una influencia más directa de las comunidades terrestres sobre las marinas habría sido la producción de arcillas y lixiviados desde los continentes (Kennedy y Wagner, 2011 y referencias incluidas), que luego serían transportados por los ríos hacia los océanos, lo que aumenta la heterogeneidad de los materiales y solutos que llegan a los ecosistemas oceánicos marginales y profundos, lo cual produciría cambios inevitables con consecuencias benéficas o perjudiciales para la vida marina. Sin embargo, también se esperaría una retención de sedimentos detríticos en tierra a través de la estabilización microbiana (trapping and binding). Por último, es probable que el lapso de tiempo desde el inicio de la vida terrestre hasta la evolución de las primeras plantas, permitiera la transformación de sustratos costeros y de los interiores continentales en sustratos orgánicos y ricos en nutrientes que más tarde serían explotados por organismos más evolucionados en la transición Neoproterozoico-Fanerozoico.

En general, la transición lógica de cianobacterias (y otras bacterias y arqueas), a algas (y protistas y hongos), a plantas no vasculares, a plantas vasculares, puede ser válida, pero la cronología de tales eventos necesita ser actualizada con la última información disponible y pertinente. La idea de que los continentes eran prácticamente estériles en el Precámbrico subestima el impacto que los microbios pudieron tener en estos ambientes. Más aún, la idea de que los primeros ecosistemas terrestres fueron dominados por plantas debe ser abandonada por completo. Esto no descarta que el advenimiento de las plantas en el Fanerozoico haya tenido efectos más profundos en el intemperismo continental, la formación del suelos profundos, y la oxigenación de la atmósfera (Labandeira, 2005; Taylor et al., 2009), pero ignorar la existencia de ecosistemas terrestres microbianos previos al Fanerozoico (posiblemente desde el Paleoarqueano), impide una comprensión realista de la evolución de la biósfera y de su influencia en la geósferaatmósfera-hidrósfera en el tiempo.

\section{Referencias}

Abed, R.M.M., Ramette, A., Hübner, V., De Dekker, P., de Beer, D., 2011, Microbial diversity of eolian dust sources from saline lake sediments and biological soil crusts in arid Southern Australia: FEMS Microbiology Ecology, 80, 294-304.

Adam, J., Rushmer, T., O'Neil, J., Francis, D., 2012, Hadean greenstones from the Nuvvuagittuq fold belt and the origin of the Earth's early continental crust: Geology, 40, 363-366.

Al-Bader, D., Eliyas, M., Rayan, R., Radwan, S., 2012, Air-dustborne associations of phototrophic and hydrocarbon-utilizing microorganisms: Promising consortia in volatile hydrocarbon bioremediation: Environmental Science and Pollution Research, 19, 3997-4005.

Aller, J.Y., Aller, R.C., Kemp, P.F., Chistoserdov, A.Y., Madrid, V.M., 2010, Fluidized muds: a novel setting for the generation of biosphere diversity through geologic time: Geobiology, 8 169-178.

Allison, P.A., Bottjer, D.J., 2011, Taphonomy: Bias and Process Through Time, in Allison, P.A., Bottjer, D.J. (eds.), Taphonomy, Process and Bias Through Time: Topics in Geobiology 32: New York, New York, USA, Springer, 1-17.

Allwood, A.C., Walter, M.R., Kamber, B.S., Marshall, C.P., Burch, I.W., 2006, Stromatolite reef from the Early Archaean Era of Australia: Nature, 441, $714-718$. 
Altermann, W., 2008, Accretion, trapping and binding of sediment in Archean stromatolites -morphological expression of the antiquity of life, in Botta, O., Bada, J., Gómez, E.J., Javaux, E., Selsis, F., Summons, R. (eds.), Strategies of Life Detection. Space Science Reviews: New York, New York, USA, Springer, 135, 55-79.

Altermann, W., Schopf, J.W., 1995, Microfossils from the Neoarchean Campbell Group, Griqualand West Sequence of the Transvaal Supergroup, and their paleoenvironmental and evolutionary implications: Precambrian Research, 75, 65-90.

Aspler, L.B., Donaldson, J.A., 1986, Paleoclimatology of Nonacho Basin (Early Proterozoic), Northwest Territories, Canada: Palaeogeography, Palaeoclimatology, Palaeoecology, 56, 17-34.

Awramik, S., 1992, The oldest records of photosynthesis: Photosynthesis Research, 33, 75-89.

Awramik, S.M., Buchheim, H.P., 2009, A Giant, Late Archean Lake System: The Meentheena Member (Tumbiana Formation; Fortescue Group), Western Australia: Precambrian Research, 174, 215-240.

Awramik, S.M., Schopf, J.W., Walter, M.R., 1983, Filamentous fossil bacteria from the Archean of Western Australia: Precambrian Research, 20, 357-374.

Bambach, R.K., 1999, Energetics in the global marine fauna: a connection between terrestrial diversification and change in the marine biosphere: Geobios, 32, 131-144.

Bamforth, S.S., 1984, Microbial distributions in Arizona deserts and woodlands: Soil Biology \& Biochemistry, 16(2), 133-137.

Bamforth, S.S., 2004, Water film fauna of microbiotic crusts of a warm desert: Journal of Arid Environments, 56, 413-423.

Bandopadhyay, P.C., Eriksson, P.G., Roberts, R.J., 2010, A vertic paleosol at the Archean-Proterozoic contact from the Singhbhum-Orissa craton, eastern India: Precambrian Research, 177, 277-290.

Banerjee, D.M., 1996, A Lower Proterozoic paleosol at BGC-Aravalli boundary in south-central Rajasthan, India: Journal of the Geological Society, 48, 277-288

Banerjee, N.R., Furnes, H., Muehlenbachs, K., Staudigel, H., de Wit, M.J., 2006, Preservation of $c a$. 3.4-3.5 Ga microbial biomarkers in pillow lavas and hyaloclastites from the Barberton Greenstone Belt, South Africa: Earth and Planetary Science Letters, 241, 707-722.

Barker, W.W., Welch, S.A., Chu, S., Banfield, J.F., 1998, Experimental observations of the effects of bacteria on aluminosilicate weathering: American Mineralogist, 83, 1551-1563.

Baross, J.A., Hoffman, S.E., 1985, Submarine hydrothermal vents and associated gradient environments as sites for the origin and evolution of life: Origins of Life, 15, 327-345.

Barton, H.A., Northup, D.E., 2007, Geomicrobiology in cave environments: past, current and future perspectives: Journal of Cave and Karst Studies, 69, 163-178

Bates, S.T., Garcia-Pichel, F., 2009, A culture-independent study of free-living fungi in biological soil crusts of the Colorado Plateau: their diversity and relative contribution to microbial biomass: Environmental Microbiology, 11, 56-67.

Battistuzzi, F.U., Hedges, S.B., 2009, A major clade of prokaryotes with ancient adaptations to life on land: Molecular and Biological Evolution, 26, 335-344.

Battistuzzi, F.U., Feijao, A., Hedges, S.B., 2004, A genomic timescale of prokaryote evolution: insights into the origin of methanogenesis, phototrophy, and the colonization of land: BMC Evolutionary Biology, 4, 1-14.

Bauld, J., 1981, Geobiological role of cyanobacterial mats in sedimentary environments: production and preservation of organic matter: BMR Journal of Australian Geology and Geophysics, 6, 307-317.

Bauld, J., D’Amelio, E., Farmer, J.D., 1992, Modern microbial mats, in Schopf, J.W., Klein, C. (eds.), The Proterozoic Biosphere: New York, New York, USA, Cambridge University Press, 261-269.

Belnap, J., Gillette, D.A., 1998, Vulnerability of desert biological soil crusts to wind erosion: the influences of crust development, soil texture, and disturbance: Journal of Arid Environments, 39, 133-142.

Belnap, J., Lange, O.L., 2001, Biological Soil Crusts: Structure, Function, and Management, Ecological Studies Series: Berlin, Germany, Springer, $510 \mathrm{p}$.
Bennett, P.C., Rogers, J.R., Choi, W.J., 2001, Silicates, silicate weathering, and microbial ecology: Geomicrobiology Journal, 18, 3-19.

Beraldi-Campesi, H., Hartnett, H.E., Anbar, A., Gordon, G.W., GarciaPichel, F., 2009, Effect of biological soil crusts on soil elemental concentrations: implications for biogeochemistry and as traceable biosignatures of ancient life on land: Geobiology, 7, 348-359.

Beraldi-Campesi, H., Farmer, J.D., Garica-Pichel, F., 2011, Evidence for Mesoproterozoic life on land and its modern counterpart in arid soils in GS Annual Meeting: Minneapolis, USA, Geological Society of America, 158 p.

Bernard, S., Benzerara, K., Beyssac, O., Menguy, N., Guyot, F., Brown, G.E. Jr., Goffe, B., 2007, Exceptional preservation of fossil plant spores in high-pressure metamorphic rocks: Earth and Planetary Science Letters, 262, 257-272.

Beukes, N.J., Dorland, H., Gutzmer, J., Nedachi, M., Ohmoto, H., 2002, Tropical laterites, life on land, and the history of atmospheric oxygen in the Paleoproterozoic: Geology, 30, 491-494.

Blackwell, M., 2000, Terrestrial life: fungal from the start?: Science, 289, 1884-1885.

Blank, C.E., 2009, Not so old Archaea - the antiquity of biogeochemical processes in the archaeal domain of life: Geobiology, 7, 495-514.

Booth, W.E., 1941, Algae As Pioneers in Plant Succession and Their Importance in Erosion Control: Ecology, 22, 38-46.

Bouvier, A. Wadhwa, M., 2010, The age of the Solar System redefined: Nature Geoscience, 3, 637-641.

Boyet, M., Carlson, R.W., 2005, 142Nd evidence for early (>4.53 Ga) global differentiation of the silicate Earth: Science, 309, 576-581.

Brady, N.C., Weil, R.R., 2008, The Nature and Properties of Soils $\left(14^{\text {th }}\right.$ Edition): Upper Saddle River, New Jersey, USA, Pearson-Prentice Hall, 992 p.

Brocks, J.J., 2011, Millimeter-scale concentration gradients of hydrocarbons in Archean shales: Live-oil escape or fingerprint of contamination?: Geochimica et Cosmochimica Acta, 75, 3196-3213.

Büdel, B., Weber, B., Kuhl, M., Pfanz, H., Sultemeyer, D., Wessels, D., 2004, Reshaping of sandstone surfaces by cryptoendolithic cyanobacteria: bioalkalisation causes chemical weathering in arid landscapes: Geobiology, 2, 261-268.

Buick, R., 2008, When did oxygenic photosynthesis evolve?: Philosophycal Transactions of the Royal Society of London, Biological Sciences, 363, 2731-2743.

Buick, R., 2010, Early life: Ancient acritarchs: Nature, 463, 885-886.

Buick, R., Thornett, J.R., McNaughton, N.J., Smith, J.B., Barley, M.E., Savage, M., 1995, Record of emergent continental crust $\sim 3.5$ billion years ago in the Pilbara Craton of Australia: Nature, 375, 574-577.

Campbell, S.E., 1979, Soil stabilization by a prokaryotic desert crust: implications for Precambrian land biota: Origins of Life, 9, 335-348.

Campbell, S.E., Seeler, J., Golubic, S., 1989, Desert crust formation and soil stabilization, in Skujins, J. (ed.) Uses of microbiological processes in arid lands for desertification control and increased productivity (UNEP). Arid Soil Research and Rehabilitation: Taylor and Francis, 3, 217-228.

Canup, R.M., Righter, K., 2000, Origin of the Earth and Moon: Tucson, Arizona, USA, University of Arizona Press, $555 \mathrm{p}$.

Catling, D.C., Zahnle, K.J., McKay, C.P., 2001, Biogenic methane, hydrogen escape, and the irreversible oxidation of early Earth: Science, 293, 839-843.

Cavosie, A.J., Valley, J.W., Wilde, S., 2007, The oldest terrestrial mineral record: A review of 4400 to 3900 Ma detrital zircons from Jack Hills, Western Australia, in van Kranendonk, M.J., Smithies, R.H., Bennett, V. (eds.), Earth's Oldest Rocks. Developments in Precambrian Geology, Series 15: Amsterdam, The Netherlands, Elsevier, 91-111.

Chanal, A., Chapon, V., Benzerara, K., Barakat, M., Christen, R., Achouak, W., Barras, F., Heulin, T., 2006, The desert of Tataouine: an extreme environment that hosts a wide diversity of microorganisms and radiotolerant bacteria: Environmental Microbiology, 8, 514-525.

Chen, Y., Wu, L., Boden, R., Hillebrand, A., Kumaresan, D., Moussard, H., Baciu, M., Lu, Y., Murrell, J.C., 2009, Life without light: microbial diversity and evidence of sulfur- and ammonium-based chemolithotrophy in Movile Cave: ISME Journal, 3, 1093-1104. 
Chenu, C., Stotzky, G., 2002, Interactions between microorganisms and soil particles: an overview, in Huang, P.M., Bollag, J.M., Senesi, N. (eds.), Interactions between Soil Particles and Microorganisms: Impact on the Terrestrial Ecosystem: John Wiley y Sons, Chichester, England Ltd. 4-40.

Chorover, J., Kretzschmar, R., Garcia-Pichel, F., Sparks, D.L., 2007, Soil biogeochemical processes within the Critical Zone: Elements 3, 321-326.

Cloud, P., Germs, A., 1971, New Pre-Paleozoic Nannofossils from the Stoer Formation (Torridonian), Northwest Scotland: Geological Society of America Bulletin, 82, 3469-3474.

Condie, K.C., 2004, Precambrian superplume events, in Eriksson, P.G., Altermann, W., Nelson, D.R., Mueller, W.U., Catuneanu, O. (eds.), The Precambrian Earth: Tempos and Events, Developments in Precambrian Geology 12: Amsterdam, The Netherlands, Elsevier, 163-173.

Corcoran, P.L., Mueller, W.U., 2004, Aggressive Archaean weathering, in Eriksson, P.G., Altermann, W., Nelson, D.R., Mueller, W.U., Catuneanu, O. (eds.), The Precambrian Earth: Tempos and Events, Developments in Precambrian Geology 12: Amsterdam, The Netherlands, Elsevier, 494-504.

Dauphas, N., van Zuilen, M., Wadhwa, M., Davis, A.M., Martey, B., Janney, P.E., 2004, Clues from $\mathrm{Fe}$ isotope variations on the origin of early Archean BIFs from Greenland: Science, 302, 2077-2080.

David, L.A., Alm, E.J., 2011, Rapid evolutionary innovation during an Archaean genetic expansion: Nature, 469, 93-96.

Davison, J., 1999, Genetic Exchange between Bacteria in the Environment: Plasmid, 42, 73-91.

Deming, D., 2002, Origin of the Ocean and Continents: A Unified Theory of the Earth: International Geology Review, 44, 137-152.

DesMarais, D.J., 2000, When did photosynthesis emerge on Earth?: Science, 289, 1703-1705.

DiMichele, W.A., Hook, R.W., 1992, Paleozoic terrestrial ecosystems, in Behrensmeyer, A.K., Damuth, J.D., DiMichele, W.A., Potts, R., Suess, H.D., Wing, S.L. (eds.), Terrestrial ecosystems through time: Chicago, Illinois, USA, Chicago University Press, 205-325.

Dong, G.R., Li, C.Z., Jin, T., Gao, S.Y., Wu, D., 1987, Some results on soil wind-tunnel imitating experiment: Chinese Science Bulletin, $32,297-301$

Dott, R.H. Jr., 2003, The importance of eolian abrasion in supermature quartz sandstones and the paradox of weathering on vegetation-free landscapes: Journal of Geology, 111, 387-405.

Driese, S.G., Gordon-Medaris, L. Jr., 2008, Evidence for biological and hydrological controls on the development of a Paleoproterozoic paleoweathering profile in the Baraboo Range, Wisconsin, USA: Journal of Sedimentary Research, 78, 443-457.

Driese, S.G., Simpson, E.L., Eriksson, K.A., 1995, Redoximorphic Paleosols in Alluvial and Lacustrine Deposits, $1.8 \mathrm{Ga}$ Lochness Formation, Mount Isa, Australia: Pedogenic Processes and Implications for Paleoclimate: Journal of Sedimentary Research, A65, 675-689.

Ehrlich, H.L., 2002, Geomicrobiology: New york, New York, USA, Marcel Dekker, 800 p.

El Albani, A., Bengtson, S., Canfield, D.E., Bekker, A., Macchiarelli, R., Mazurier, A., Hammarlund, E.U., Boulvais, P., Dupuy, J.J., Fontaine, C., Fürsich, F.T., Gauthier-Lafaye, F., 2010, Large colonial organisms with coordinated growth in oxygenated environments $2.1 \mathrm{Gyr}$ ago: Nature, 466, 100-104.

Elbert, W., Weber, B., Burrows, S., Steinkamp, J., Büdel, B., Andreae, M.O., Pöschl, U., 2012, Contribution of cryptogamic covers to the global cycles of carbon and nitrogen: Nature Geoscience, 5, 459-462.

Engel, A.S., 2010, Microbial diversity of cave ecosystems, in Barton, L., Mandl, M., Loy, A. (eds.), Geomicrobiology: Molecular y Environmental Perspectives: Albuquerque, New Mexico, USA, Springer, 219-238.

Eriksson, P.G., Martins-Neto, M.A., 2004, Commentary, in Eriksson, P.G., Altermann, W., Nelson, D.R., Mueller, W.U., Catuneanu, O. (eds.), The Precambrian Earth: Tempos and Events, Developments in Precambrian Geology 12: Amsterdam, The Netherlands, Elsevier,
$677-680$.

Eriksson, P.G., Simpson, E.L., Eriksson, K.A., Bumby, A.J., Steyn, G.L., Sarkar, S., 2000, Muddy roll-up structures in siliciclastic interdune beds of the c. $1.8 \mathrm{Ga}$ Waterberg Group, South Africa: PALAIOS, $15,177-183$.

Eriksson, P.G., Altermann, W., Nelson, D.R., Mueller, W.U., Catuneanu, O., 2004, The Precambrian Earth: Tempos and Events, Developments in Precambrian Geology 12: Amsterdam, The Netherlands, Elsevier, $966 \mathrm{p}$.

Farmer, J., 1999, Taphonomic modes in microbial fossilization, in Space Studies Board, Size limits of very small microorganisms, National Research Council: National Academy Press, 94-102.

Farrow, C.E., Mossman, D.J., 1988, Geology of Precambrian paleosols at the base of the Huronian supergroup, Elliot lake, Ontario, Canada: Precambrian Research, 42, 107-139.

Fedo, C.M., Whitehouse, M.J., 2002, Metasomatic origin of quartzpyroxene rock, Akilia, Greenland, and implications for Earth's earliest life: Science, 296, 1448-1452.

Fierer, N., Nemergut, D., Knight, R., Craine, J.M., 2010, Changes through time: integrating microorganisms into the study of succession: Research in Microbiology, 161, 635-642.

Fleming, E.D., Castenholz, R.W., 2007, Effects of periodic desiccation on the synthesis of the UV-screening compound, scytonemin, in cyanobacteria: Environmental Microbiology, 9, 1448-1455.

Fletcher, J.E., Martin, W.P., 1948, Some effects of algae and mold in the rain-crust of desert soils: Ecology, 29, 95-100.

Fliegel, D., Wirth, R., Simonetti, A., Furnes, H., Staudigel, H., Hanski, E., Muehlenbachs, K., 2010, Septate-tubular textures in 2.0-Ga pillow lavas from the Pechenga Greenstone Belt: a nano-spectroscopic approach to investigate their biogenicity: Geobiology, 8, 372-390.

Franz, G., Mosbrugger, V., Menge, R., 1991, Carbo-Permian pteridophyll leaf fragments from an amphibolite facies basement, Tauern Window, Austria: Terra Nova, 3, 137-141.

Friedmann, E.I., 1980, Endolithic microbial life in hot and cold deserts: Origins of Life, 10, 223-235.

Fritsch, F.E., 1922, The terrestrial alga: The Journal of Ecology, 10, 220-236.

Furnes, H., Banerjee, N.R., Muehlenbachs, K., Staudigel, H., de Wit, M., 2004, Early life recorded in Archean pillow lavas: Science, 304, $578-81$.

Furnes, H., de Wit, M., Staudigel, H., Rosing, M., Muehlenbachs, K., 2007a, Vestige of Earth's oldest ophiolite: Science, 315, 1704-1707.

Furnes, H., Banerjee, N.R., Staudigel, H., Muehlenbachs, K., McLoughlin, N., de Wit, M., van Kranendonk, M., 2007b, Comparing petrographic signatures of bioalteration in recent to Mesoarchean pillow lavas: Tracing subsurface life in oceanic igneous rocks: Precambrian Research, 158, 156-176.

Gadd, G.M., 2006, Fungi in biogeochemical cycles: Cambridge University Press, Cambridge, U.K., 490p.

Gall, Q., 1994, The Proterozoic Thelon paleosol, Northwest Territories, Canada: Precambrian Research, 68, 115-137.

Gao, Q., Garcia-Pichel, F., 2011, Microbial ultraviolet sunscreens: Nature Reviews, Microbiology, 9, 791-802.

Garcia-Pichel, F., 1998, Solar ultraviolet and the evolutionary history of cyanobacteria: Origins of Life and Evolution of the Biosphere, 28, 321-347.

Garcia-Pichel, F., Castenholz, R.W., 1991, Characterization and biological implications of scytonemin, a cyanobacterial sheath pigment: Journal of Phycology, 27, 395-409.

Garcia-Pichel, F., Pringault, O., 2001, Cyanobacteria track water in desert soils: Nature, 413, 380-381.

Garcia-Pichel, F., Wojciechowski, M.F., 2009, The evolution of a capacity to build supra-cellular ropes enabled filamentous cyanobacteria to colonize highly erodible substrates: PLoS ONE, 4, e7801.

Garcia-Pichel, F., Lopez-Cortes, A., Nubel, U., 2001, Phylogenetic and morphological diversity of cyanobacteria in soil desert crusts from the Colorado Plateau: Applied Environmental Microbiology, 67, 1902-1910. 
Garcia-Pichel, F., Belnap, J., Neuer, S., Schanz, F., 2003, Estimates of global cyanobacterial biomass and its distribution: Cyanobacterial Research 4: Archive for Hydrobiology/Algological Studies, 109, 213-227.

García-Ruiz, J.M., Carnerup, A., Christy, A.G., Welham, N.J., Hyde, S.T., 2002, Morphology: an ambiguous indicator of biogenicity: Astrobiology, 2 (2), 353-369.

García-Ruiz, J.M., Hyde, S.T., Carnerup, A.M., Christy, A.G., van Kranendonk, M.J., Welham, N.J., 2003, Self-assembled silicacarbonate structures and detection of ancient microfossils: Science, 302, 1194-1197.

Gaucher, C., Sial, A.N., Halverson, G.P., Frimmel, H.E., 2010, Neoproterozoic-Cambrian Tectonics, Global Change and Evolution: a Focus on Southwestern Gondwana, Developments in Precambrian Geology 16: Amsterdam, The Netherlands, Elsevier, 498 p.

Gay, A.L., Grandstaff, D.E., 1980, Chemistry and mineralogy of Precambrian paleosols at Elliot Lake, Ontario, Canada: Precambrian Research, 12, 349-373.

Geesey, G., Jang, L., 1990, Extracellular polymers for metal binding, in Ehrlich, H.L., Brierley, C.L. (eds.), Microbial Mineral Recovery: New York, New York, USA, McGraw-Hill, 223-249.

Gensel, P.G., 2008, The earliest land plants: Annual Review of Ecology and Evolutionary Systems, 39, 459-477.

Ghiorse, W., Wilson, J., 1988, Microbial ecology of the terrestrial subsurface, in Laskin, A. (ed.), Advances in Applied Microbiology: New York, New York, USA, Academic Press, 107-172.

Giordanino, F., Strauch, S.M., Villafañe, V.E., Helbling, E.W., 2011 Influence of temperature and UVR on photosynthesis and morphology of four species of cyanobacteria: Journal of Photochemistry and Photobiology, B: Biology, 103, 68-77.

Glass, J.B., Wolfe-Simon, F., Anbar, A.D., 2009, Coevolution of metal availability and nitrogen assimilation in cyanobacteria and algae: Geobiology, 7, 100-123.

Glikson, A., 2007, Early Archean asteroid impacts on Earth: stratigraphic and isotopic age correlations and possible geodynamic consequences, in van Kranendonk, M.J., Smithies, R.H., Bennett, V. (eds.), Earth's Oldest Rocks. Developments in Precambrian Geology, Series 15: Amsterdam, The Netherlands, Elsevier, 1087-1103.

Glover, J.F., Kah, L.C., 2006, Speleothem deposits in a Proterozoic paleokarst, Mesoproterozoic dismal lakes group, Arctic Canada Geological Society of America Southeastern Section, 55 ${ }^{\text {th }}$ Annual Meeting: Knoxville, Tennessee, USA, Geological Society of America Abstracts with Programs, 38, 36.

Gogarten, J.P., Fournier, G., Zhaxybayeva, O., 2007, Gene Transfer and the Reconstruction of Life's Early History from Genomic Data, in Botta, O., Bada, J.L., Gomez-Elvira, J., Javaux, E., Selsis, F., Summons, R. (eds.), Strategies of Life Detection. Space Science Series of ISSI: Amsterdam, The Netherlands, Springer, 115-131.

Golubic, S., Hofmann, H.J., 1976, Comparison of modern and midPrecambrian Entophysalidaceae (Cyanophyta) in stromatolitic algal mats: cell division and degradation: Journal of Paleontology, 50, 1074-1082.

Golubic, S., Campbell, S.E., 1979, Analagous microbial forms in Recent subaerial habitats and in Precambrian cherts: Gloethece coerulea Geitler and Eosynechococcus moorei Hoffmann: Precambrian Research, 8, 201-217.

Golubic, S., Seong-Joo, L., 1999, Early Cyanobacterial fossil record: preservation, palaeoenvironments and identification: European Journal of Phycology, 34, 339-348.

Gomes, R., Levison, H.F., Tsiganis, K., Morbidelli, A., 2005, Origin of the cataclysmic late heavy bombardment period of the terrestrial planets: Nature, 435, 466-469.

Gradzinski, M., Chmiel, M.J., Lewandowska, A., Michalska-Kasperkiewicz, B., 2010, Siliciclastic micro-stromatolites in a sandstone cave: role of trapping and binding of detrital particles in formation of cave deposits. Annales Societatis Geologorum Poloniae, 80, 303-314

Grandstaff, D.E., Edelman, M.J., Foster, R.W., Zbinden, E., Kimberley, M.M., 1986, Chemistry and mineralogy of Precambrian paleosols at the base of the Dominion and Pongola Groups: Precambrian Research, 32, 97-131.

Gray, J., Shear, W.A., 1992, Early life on land: American Scientist, 80, 444-456.

Gray, J., Boucot, A.J., 1994, Early Silurian nonmarine animal remains and the nature of the early continental ecosystem: Acta Palaeontologica Polonica, 38 (3-4), 303-328.

Gunatilaka, A., 1975, Some aspects of the biology and sedimentology of laminated algal mats from Mannar Lagoon, northwest Ceylon: Sedimentary Geology, 14, 275-300.

Gutzmer, J., Beukes, N.J., 1998, Earliest laterites and possible evidence for terrestrial vegetation in the Early Proterozoic: Geology, 26, 263-266.

Hallbauer, D.K., 1975, The plant origin of the Witwatersrand carbon. Mineral Science Engeneering, 7 (2), 111-131.

Hallbauer, D.K., van Warmelo, K.T., 1974, Fossilized plants in thucholite from Precambrian rocks of the Witwatersrand, South Africa: Precambrian Research, 1, 199-212.

Hamblin, W.K., Christiansen, E.H., 2007, Earth's Dynamic Systems (10 ${ }^{\text {th }}$ Edition), Upper Saddle River, N.J., Prentice Hall, 816 p.

Han, T.M., Runnegar, B., 1992, Megascopic eukaryotic algae from the 2.1-billion-year-old negaunee iron-formation, Michigan: Science, 257, 232-235.

Hanel, M., Montenari, M., Kalt, A., 1999, Determining sedimentation ages of high-grade metamorphic gneisses by their palynological record: a case study in the northern Schwarzwald (Variscan Belt, Germany): International Journal of Earth Sciences, 88, 49-59.

Harrison, S.P., Kohfeld, K.E., Roelandt, C., Claquin, T., 2001, The role of dust in climate changes today, at the last glacial maximum and in the future: Earth Sciences Review, 54, 43-80.

Hartmann, W.K., Ryder, G., Dones, L., Grinspoon, D., 2000, The timedependent intense bombardment of the primordial Earth/Moon system, in Canup, R.M., Righter, K. (eds.), Origin of the Earth and Moon: Tucson, Arizona, University of Arizona Press, 493-512.

Hazen, R.M., Sverjensky, D.A., 2010, Mineral surfaces, geochemical complexities, and the origins of life, in Deamer, D., Szostak, J.W. (eds), The origins of life, volume 5, $2^{\text {nd }}$ edition: Cold Spring Harbor, Cold Spring Harbor Laboratory Press, 1-21.

Hazen, R.M., Golden, J., Downs, R.T., Hystad, G., Grew, E.S., Azzolini, D., Sverjensky, D.A., 2012, Mercury (Hg) mineral evolution: A mineralogical record of supercontinent assembly, changing ocean geochemistry, and the emerging terrestrial biosphere: American Mineralogist, 97, 1013-1042.

Heckman, D.S., Geiser, D.M., Eidell, B.R., Stauffer, R.L., Kardos, N.L., Hedges, S.B., 2001, Molecular evidence for the early colonization of land by fungi and plants: Science, 293, 1129-1133.

Herrero, A., Flores, E., 2008, The Cyanobacteria: Molecular Biology, Genomics and Evolution: Norfolk, UK, Caister Academic Press, $484 \mathrm{p}$.

Hickman, A.H., van Kranendonk, M.J., 2012, Early Earth evolution: evidence from the 3.5-1.8 Ga geological history of the Pilbara region of Western Australia: Episodes, 35, 283-297.

Hoashi, M., Bevacqua, D.C., Otake, T., Watanabe, Y., Hickman, A.H., Utsunomiya, S., Ohmoto, H., 2009, Primary haematite formation in an oxygenated sea 3.46 billion years ago: Nature Geoscience, 2, 301-306.

Hoffman, P.F., 1995, The oldest terrestrial landscape: Nature, 375, 537-538.

Hoffman, P.F., Schrag, D.P., 2002, The snowball Earth hypothesis: testing the limits of global change: Terra Nova, 14, 129-155.

Hofmann, H.J., 1976, Precambrian microflora, Belcher Islands, Canada; significance and systematics: Journal of Paleontology, 50, 10401073.

Holland, H.D., 1984, The Chemical Evolution of the Atmosphere and Oceans: Princeton, New Jersey, USA, Princeton University Press, 582 p.

Holland, H.D., 1992, Distribution and paleoenvironment interpretation of Proterozoic paleosols, in Schopf, J.W., Klein, C. (eds.), The Proterozoic Biosphere: New York, New York, USA, Cambridge University Press, 153-155. 
Holm, N.G., 1992, Marine hydrothermal systems and the origins of life: Origins of Life and Evolution of the Biosphere, 22, 181-242.

Hoppert, M., Reimer, R., Kemmling, A., Schröder, A., Günzl, B., Heinken, T., 2004, Structure and reactivity of a biological soil crust from a xeric sandy soil in central Europe: Geomicrobiology Journal, 21, 183-191.

Horodyski, R.J., Knauth, P.L., 1994, Life on land in the Precambrian: Science, 263, 494-498.

Hu, C., Liu, Y., Song, L., Zhang, D., 2002, Effect of desert soil algae on the stabilization of fine sands: Journal of Applied Phycology, 14, 281-292.

Hupe, P., 1952, Sur des problematica du Precambrien III. Division des Mines et de la Géologie: Service Géologique de Morocco, Notes et Memoires, 103, 297-383.

Iizuka, T., Horie, K., Komiya, T., Maruyama, S., Hirata, T., Hidaka, H., Windley, B.F., 2006, 4.2 Ga zircon xenocryst in an Acasta gneiss from northwestern Canada: Evidence for early continental crust: Geology, 34, 245-248.

Ishizuka, H., 2008, Protoliths of the Napier Complex in Enderby Land, East Antarctica; an overview and implication for crustal formation of Archean continents: Journal of Mineralogical and Petrological Sciences, 103, 218-225.

Jackson, T.A., 1967, Fossil Actinomycetes in Middle Precambrian Glacial Varves: Science, New Series, 155, 1003-1005.

Javaux, E.J., Marshall, C.P., Bekker, A., 2010, Organic-walled microfossils in 3.2-billion-year-old shallow-marine siliciclastic deposits: Nature, $463,934-938$.

Jickells, T.D., An, Z.S., Andersen, K.K., Baker, A.R., Bergametti, G., Brooks, N., Cao, J.J., Boyd, P.W., Duce, R.A., Hunter, K.A., Kawahata, H., Kubilay, N., LaRoche, J., Liss, P.S., Mahowald, N., Prospero, J.M., Ridgwell, A.J., Tegen, I., Torres, R., 2005, Global iron connections between desert dust, ocean biogeochemistry, and climate: Science, 308, 67-71.

Johnson, I., Watanabe, Y., Stewart, B.. Ohmoto, H., 2009, Earth's oldest $(\sim 3.4 \mathrm{Ga})$ lateritic paleosol in the Pilbara Craton, Western Australia in Goldschmidt Conference: Davos, Switzerland, Goldschmidt Conference Abstracts, A601.

Johnson, I., Watanabe, Y., Stewart, B., Ohmoto, H., 2010, Evidence for terrestrial life and an O2-rich atmosphere in the oldest ( 3.4 Ga) paleosol in the east Pilbara craton, Western Australia in $6^{\text {th }}$ Astrobiology Sci. Conference: League City, Texas, USA, Lunar and Planetary Institute, 5424

Kandler, O., 1994, The early diversification of life, in Bengston, S. (ed.), Early life on earth: New York, New York, USA, Columbia University Press, $152-161$.

Kappler, A., Pasquero, C., Konhauser, K.O., Newman, D.K., 2005, Deposition of banded iron formations by anoxygenic phototrophic $\mathrm{Fe}(\mathrm{II})$-oxidizing bacteria: Geology, 33, 865-868.

Kasting, J., 2009, How to Find a Habitable Planet: Princeton, New Jersey, USA, Princeton University Press, $326 \mathrm{p}$.

Kato, Y., Suzuki, K., Nakamura, K., Hickman, A.H., Nedachi, M., Kusakabe, M., Bevacqua, D.C., Ohmoto, H., 2009, Hematite formation by oxygenated groundwater more than 2.76 billion years ago: Earth and Planetary Science Letters, 278, 40-49.

Keller, C.K., Wood, B.D., 1993, Possibility of chemical weathering before the advent of vascular land plants: Nature, 364, 223-225.

Kendall, B., Reinhard, C.T., Lyons, T.W., Kaufman, A.J., Poulton, S.W., Anbar,A.D., 2010, Pervasive oxygenation along late Archaean ocean margins: Nature Geoscience, 3, 647-652.

Kennedy, M.J., Wagner, T., 2011, Clay mineral continental amplifier for marine carbon sequestration in a greenhouse ocean: Proceedings of the National Academy of Sciences USA, 108, 9776-9781.

Kennedy, M.J., Chadwick, O.A., Vitousek, P.M., Derry, L.A., Hendricks, D.M., 1998, Replacement of weathering with atmospheric sources of base cations during ecosystem development, Hawaiian Islands: Geology, 26, 1015-1018.

Kennedy, M., Droser, M., Mayer, L.M., Pevear, D., Mrofka, D., 2006, Late Precambrian Oxygenation; Inception of the Clay Mineral Factory:
Science, 311, 1446-1449.

Kimberley, M.M., Grandstaff, D.E., 1986, Profiles of elemental concentrations in Precambrian paleosols on basaltic and granitic parent materials: Precambrian Research, 32, 133-154.

Kirschvink, J.L., Kopp, R.E., 2008, Palaeoproterozoic ice houses and the evolution of oxygen-mediating enzymes: the case for a late origin of photosystem II: Philosophical Transaction of the Royal Society of London, Biology, 363, 2755-2765.

Klein, C., Beukes, N.J., Schopf, J.W., 1987, Filamentous microfossils in the early Proterozoic Transvaal supergroup: Their morphology, significance, and paleoenvironmental setting: Precambrian Research, $36,81-94$.

Knauth, L.P., Kennedy, M.J., 2009, The late Precambrian greening of the Earth: Nature, 460, 728-732.

Knoll, A.H., Javaux, E.J., Hewitt, D., Cohen, P., 2006, Eukaryotic organisms in Proterozoic oceans. Philosophical Transactions of the Royal Society of London, B Biological Sciences, 361: 1023-1038.

Knoll, A., Canfield, D., Konhauser, K., 2012, Fundamentals of Geobiology: Hoboken, New Jersey, USA, Blackwell Publishing Ltd., 480 p.

Koehler, I., Konhauser, K.O., Kappler, A., 2010, Role of microorganisms in Banded Iron Formations, in Barton, L., Mandl, M., Loy, A. (eds.), Geomicrobiology: Molecular and Environmental Perspective, Springer, 309-324.

Komiya, T., Maruyama, S., Nohda, S., Masuda, T., Hayashi, M., Okamoto, S., 1999, Plate tectonics at $3.8-3.7 \mathrm{Ga}$; Field evidence from the Isua accretionary complex, southern West Greenland: Journal of Geology, 107, 515-554.

Konhauser, K., 2007, Introduction to Geomicrobiology: Oxford, United Kingdom, Blackwell Publishing, $400 \mathrm{p}$.

Konhauser, K.O., Schultzelam, S., Ferris, F.G., Fyfe, W.S., Longstaffe, F.J., Beveridge, T.J., 1994, Mineral precipitation by epilithic biofilms in the Speed River, Ontario, Canada: Applied and Environmental Microbiology, 60, 549-553.

Konhauser, K.O., Newman, D.K., Kappler, A., 2005, The potential significance of microbial $\mathrm{Fe}(\mathrm{III})$ reduction during deposition of Precambrian banded iron formations: Geobiology, 3, 167-177.

Kositcin, N., Krapez, B., 2004, SHRIMP U-Pb detrital zircon geochronology of the late Archean Witwatersrand Basin, relation between zircon provenance age spectra and basin evolution: Precambrian Research, $129,141-168$.

Labandeira, C.C., 2005, Invasion of the continents: cyanobacterial crusts to tree-inhabiting arthropods: Trends in Ecology and Evolution, $20,253-262$.

Lalonde, K., Mucci, A., Ouellet, A., Gélinas, Y., 2012, Preservation of organic matter in sediments promoted by iron: Nature, 483(7388), 198-200.

Lannerbro, R., 1954, Description of some structures, possibly fossils, in Jotnian sandstone from Mångsbodarna in Dalecarlia: Geologiska Föreningens i Stockholm Förhandlingar, 76, 46-50.

Lau, K.M., Kim, M.K., 2006, Asian monsoon anomalies induced by aerosol direct effects: Climate Dynamics, 26, 855-864.

Lazcano, A., Miller, S.L., 1994, How long did it take for life to begin and evolve to cyanobacteria?: Journal of Molecular Evolution, 39, $546-554$.

Li, W., Johnson, C.M., Beard, B.L., 2012, U-Th-Pb isotope data indicate Phanerozoic age for oxidation of the $3.4 \mathrm{Ga}$ Apex Basalt: Earth and Planetary Science Letters, 319-320, 197-206.

Liu, Y.Z., Dong, G.R., Li, C.Z., 1994, A study on the factors influencing soil erosion through wind tunnel experiments: Chinese Journal of Arid Land Research, 7, 359-367.

Lopez-Garcia, P., Moreira, D., Douzery, E.J.P., Forterre, P., van Zuilen, M., 2006, Ancient fossil record and early evolution (ca. 3.8 to $0.5 \mathrm{Ga}$ ), in Gargaud, M., Claeys, P., López-García, P., Martin, H., Montmerle, T., Pascal, R., Reisse, J. (Eds.), From Suns to Life: a multidisciplinary approach to the history of life on Earth: Earth, Moon, and Planets, Springer, 247-290.

Lowe, D.R., 1980, Stromatolites 3,400-Myr old from the Archean of Western Australia: Nature, 284, 441-443. 
Lozupone, C.A., Knight, R., 2007, Global patterns in bacterial diversity: Proceeding of the National Academy of Sciences USA, 104, 11436-11440.

Lyons, T.W., Gill, B.C., 2010, Ancient Sulfur Cycling and Oxygenation of the Early Biosphere: Elements, 6, 93-99.

Macfarlane, A.W., Danielson, A., Holland, H.D., 1994, Geology and major and trace element chemistry of the late Archean weathering profiles in the Fortescue Group, Western Australia: Implications for atmospheric $\mathrm{pO}_{2}$ : Precambrian Research, 65, 297-317.

Madigan, M.T., Marrs, B.L., 1997, Extremophiles: Scientific American, 276(4), 66-71.

Madigan, M., Martinko, J., Parker, J., 2003, Brock: Biology of Microorganisms ( $10^{\text {th }}$ Edition): Upper Saddle River, New Jersey, USA, Pearson-Prentice Hall, $1061 \mathrm{p}$.

Malam Issa, O., Le Bissonnais, Y., Défarge, C., Trichet, J., 2001, Role of a cyanobacterial cover on structural stability of sandy soils in the Sahalian part of western Niger: Geoderma, 101, 15-30.

Martini, J.E.J., 1994, A late Archaean-Palaeoproterozoic (2.6 Ga) palaeosol on ultramafics in the eastern Transvaal, South Africa: Precambrian Research, 67, 159-180.

Mazor, G., Kidron, G.J., Vonshak, A., Abeliovich, A., 1996, The role of cyanobacterial exopolysaccharides in structuring desert microbial crusts: FEMS Microbiology Ecology, 21, 121-130.

McConnell, R.L., 1974, Preliminary report of microstructures of probable biologic origin from the Mescal Formation (Proterozoic) of central Arizona: Precambrian Research, 1, 227-234.

McKeegan, K.D., Kudryavtsev, A.B., Schopf, J.W., 2007, Raman and ion microscopic imagery of graphitic inclusions in apatite from older than 3830 Ma Akilia supracrustal rocks, west Greenland: Geology, $35,591-594$.

McTainsh, G.H., Strong, C.L., 2007, The role of aeolian dust in ecosystems: Geomorphology - Special Issue on Geomorphology and Ecosystems, $89,39-54$.

Meadow, J.F., Zabinski, C.A., 2012, Spatial heterogeneity of eukaryotic microbial communities in an unstudied geothermal diatomaceous biological soil crust: Yellowstone National Park, WY, USA: FEMS Microbiology Ecology, 82(1), 182-191.

Mloszewska, A.M., Pecoits, E., Cates, N.L., Mojzsis, S.J., O'Neil, J., Robbins, L.J., Konhauser, K.O., 2012, The composition of Earth's oldest iron formations: The Nuvvuagittuq Supracrustal Belt (Quebec, Canada): Earth and Planetary Science Letters, 317-318, 331-342.

Mojzsis, S.J., Harrison, M.T., Pidgeon, R.T., 2001, Oxygen-isotope evidence from ancient zircons for liquid water at the Earth's surface 4,300 Myr ago: Nature, 409, 178-181.

Mossman, D.J., Minter, W.E.L., Dutkiewicz, A., Hallbauer, D.K., George, S.C., Hennigh, Q., Reimer, T.O., Horscroft, F.D., 2008, The indigenous origin of Witwatersrand "carbon": Precambrian Research, 164, 173-186.

Mulkidjanian, A.Y., Bychkovc, A.Y., Dibrova, D.V., Galperin, M.Y., Koonin, E.V., 2012, Origin of the first cells at terrestrial, anoxic geothermal fields: Proceedings of the National Academy of Sciences USA, 109, E821-E830.

Myers, J.S., 2001, Protoliths of the 3.8-3.7Ga Isua Greenstone Belt, West Greenland: Precambrian Research, 105, 129-141.

Myers, J.S., 2004, Isua enigmas: illusive tectonic, sedimentary, volcanic and organic features of the $>3.7 \mathrm{Ga}$ Isua greenstone belt, southwest Greenland, in Eriksson, P.G., Altermann, W., Nelson, D.R., Mueller, W.U., Catuneanu, O. (eds.), The Precambrian Earth: Tempos and Events, Developments in Precambrian Geology 12: Amsterdam, The Netherlands, Elsevier, 66-73.

Nagy, M., Perez, A., Garcia-Pichel, F., 2005, The prokaryotic diversity of biological soil crusts in the Sonoran desert (Organ Pipe Cactus National Monument, AZ): FEMS Microbiology Ecology, 54, 233-245.

Neher, D.A., Lewins, S.A., Weicht, T.R., Darby, B.J., 2009, Microarthropod communities associated with biological soil crusts in the Colorado Plateau and Chihuahuan deserts: Journal of Arid Environments, $73,672-677$.
Nelson, D.R., 2004, Earth's formation and first billion years, in Eriksson, P.G., Altermann, W., Nelson, D.R., Mueller, W.U., Catuneanu, O. (eds.), The Precambrian Earth: Tempos and Events, Developments in Precambrian Geology 12: Amsterdam, The Netherlands, Elsevier, $3-27$.

Nemergut, D.R., Costello, E.K., Hamady, M., Lozupone, C., Jiang, L., Schmidt, S.K., Fierer, N., Townsend, A.R., Cleveland, C.C., Stanish, L., Knight, R., 2011, Global patterns in the biogeography of bacterial taxa: Environmental Microbiology. 13, 135-144.

Nesbitt, H.W., Young, G.M., 2004, Aggressive Archaean weathering, in Eriksson, P.G., Altermann, W., Nelson, D.R., Mueller, W.U., Catuneanu, O. (eds.), The Precambrian Earth: Tempos and Events, Developments in Precambrian Geology 12: Amsterdam, The Netherlands, Elsevier, 482-493.

Noffke, N., 2010, Geobiology: microbial mats in sandy deposits from the Archean era to today: Springer-Verlag, Berlin, 194p.

Noffke, N., Gerdes, G., Klenke, T., Krumbein, W., 2001, Perspectives. Microbially Induced Sedimentary Structures - a new category within the classification of primary sedimentary structures: Journal of Sedimentary Research, 71, 649-656.

Noffke, N., Eriksson, K.A., Hazen, R.M., Simpson, E.L., 2006, A new window into Early Archean life: microbial mats in Earth's oldest siliciclastic tidal deposits (3.2 Ga Moodies Group, South Africa): Geology 34(4), 253-256.

Noffke, N., Christian, D.R., Hazen, R.M., 2011, A (cyano-)bacterial ecosystem in the Archean 3.49 Ga Dresser Formation, Pilbara, Western Australia. GSA Annual Meeting in Minneapolis. Paper No. 56-11: Geological Society of America Abstracts with Programs, $43,159$.

Nutman, A.P., 2006, Antiquity of the oceans and continents: Elements, 2, 223-227.

Nutman, A.P., McGregor, V.R., Friend, C.R.L., Bennett, V.C., Kinny, P.D., 1996, The Itsaq Gneiss Complex of southern West Greenland; the world's most extensive record of early crustal evolution (3900-3600 Ma): Precambrian Research, 78, 1-39.

Nutman, A.P., Friend, C.R.L., Bennett, V.C., 2002, Evidence for 3650-3600 Ma assembly of the northern end of the Itsaq Gneiss Complex, Greenland: Implication for early Archaean tectonics: Tectonics, 21, 1-28.

Nutman, A.P., Friend, C.R.L., Bennett, V.C., Wright, D., Norman, M.D., 2010, $\geq 3700$ Ma pre-metamorphic dolomite formed by microbial mediation in the Isua supracrustal belt (W. Greenland), Simple evidence for early life?: Precambrian Research, 183, 725-737.

Och, L.M., Shields-Zhou, G.A., 2012, The Neoproterozoic oxygenation event: Environmental perturbations and biogeochemical cycling: Earth Science Reviews, 110, 26-57.

Ohmoto, H., 1996, Evidence in pre-2.2 Gapaleosols for the early evolution of atmospheric oxygen and terrestrial biota: Geology, 24, 1135-1138.

Ohmoto, H., 2004, Archean atmosphere, hydrosphere, and biosphere, in Eriksson, P.G., Altermann, W., Nelson, D.R., Mueller, W.U., Catuneanu, O. (eds.), The Precambrian Earth: Tempos and Events, Developments in Precambrian Geology 12: Amsterdam, The Netherlands, Elsevier, 361-388.

Omelon, C.R., Pollard, W.H., Ferris, F.G., 2006, Chemical and ultrastructural characterization of high arctic cryptoendolithic habitats: Geomicrobiology Journal, 23, 189-200.

O'Neil, J., Carlson, R.W., Francis, D., Stevenson, R.K., 2009, Response to Comment on "Neodymium-142 Evidence for Hadean Mafic Crust": Science, 325, 267b.

O'Neil, J., Francis, D., Carlson, R.W., 2011, Implications of the Nuvvuagittuq Greenstone Belt for the formation of Earth's early crust: Journal of Petrology, 52, 985-1009.

Pace, N., 1997, A Molecular View of Microbial Diversity and the Biosphere: Science, 276, 734-740.

Palmer, J.A., Phillips, G.N., McCarthy, T.S., 1989, Paleosols and their relevance to Precambrian atmospheric composition: Journal of Geology, 97, 77-92. 
Pandit, M.K., de Wall, H., Chauhan, N.K., 2008, Paleosol at the ArcheanProterozoic contact in NW India revisited: Evidence for oxidizing conditions during paleo-weathering?: Journal of Earth Systems Science, 117, 201-209.

Papineau, D., DeGregorio, B.T., Cody, G.D., Fries, M.D., Mojzsis, S.J., Steele, A., Stroud, R.M., Fogel, M.L., 2010a, Ancient graphite in the Eoarchean quartz-pyroxene rock from Akilia in southwest Greenland I: Petrographic and spectroscopic characterization: Geochimica et Cosmochimica Acta, 74, 5862-5883.

Papineau, D., DeGregorio, B.T., Stroud, R.M., Steele, A., Pecoits, E., Konhauser, K., Wang, J., Fogel, M.L., 2010b, Ancient graphite in the Eoarchean quartz-pyroxene rock from Akilia in southern West Greenland II: Isotopic and chemical compositions and comparison with Paleoproterozoic banded iron formations: Geochimica et Cosmochimica Acta, 74, 5884-5905.

Papineau, D., De Gregorio, B.T., Cody, G.D., O’Neil, J., Steele, A., Stroud, R.M., Fogel, M.L., 2011, Young poorly crystalline graphite in the 3.8-Gyr-old Nuvvuagittuq banded iron formation: Nature Geoscience, 4, 376-379.

Paul, E.A., Collins, H.P., Leavitt, S.W., 2001, Dynamics of resistant soil carbon of Midwestern agricultural soils measured by naturally occurring 14C abundance: Geoderma, 104, 239-256.

Porter, M.L., Engel, A.S., Kinkle, B., Kane, T.C., 2009, Productivitydiversity relationships from chemolithoautotrophically based sulfidic karst systems: International Journal of Speleology, 38, 27-40.

Potts, M., 1999, Mechanisms of desiccation tolerance in cyanobacteria: European Journal of Phycology, 34, 319-328.

Potts, M., Friedmann, E.I., 1981, Effects of water stress on cryptoendolithic cyanobacteria from hot desert rocks: Archives of Microbiology, 130, 267-271.

Prasad, N., Roscoe, S.M., 1996, Evidence of anoxic to oxic atmospheric change during 2.45-2.22 Ga from lower and upper sub-Huronian paleosols, Canada: Catena, 27, 105-121.

Prave, A.R., 2002, Life on land in the Proterozoic: Evidence from the Torridonian rocks of northwest Scotland: Geology, 30, 811-814.

Pufahl, P.K., Hiatt, E.E., 2012, Oxygenation of the Earth's atmosphereocean system: a review of physical and chemical sedimentologic responses: Journal of Marine and Petroleum Geology, 32, 1-20.

Rasmussen, B., 2000, Filamentous microfossils in a 3,235-million-yearold volcanogenic massive sulphide deposit: Nature, 405, 676-679.

Rasmussen, B., Fletcher, I.R., Brocks, J.J., Kilburn, M.R., 2008, Reassessing the first appearance of eukaryotes and cyanobacteria: Nature, 455, 1101-1104.

Rasmussen, B., Blake, T.S., Fletcher, I.R., Kilburn, M.R., 2009, Evidence for microbial life in synsedimentary cavities from 2.75 Ga terrestrial environments: Geology, 37, 423-426.

Reddy, S.G., Garcia-Pichel, F., 2006, The community and phylogenetic diversity of biological soil crusts in the Colorado Plateau studied by molecular fingerprinting and intensive cultivation: Microbial Ecology, 52, 345-357.

Reimer, T.O., 1986, Alumina-rich rocks from the Early Precambrian of the Kaapvaal craton as indicators of paleosols and as products of other decompositional reactions: Precambrian Research, 32, 155-179.

Retallack, G.J., 1986a, The fossil record of soils, in Wright, P.V. (ed.), Paleosols: their recognition and interpretation: Oxford, United Kingdom, Blackwells, 1-57.

Retallack, G.J., 1986b, Reappraisal of a 2200 Ma-old paleosol near Waterval Onder, South Africa: Precambrian Research, 32, 195-232.

Retallack, G.J., 2001, Soils of the Past: An introduction to Paleopedology ( $2^{\text {nd }}$ Edition): Oxford, United Kingdom, Blackwell Science, Ltd., $404 \mathrm{p}$.

Retallack, G.J., 2009, Cambrian-Ordovician non-marine fossils from South Australia: Alcheringa. 33, 355-391.

Retallack, G.J, 2011, Criteria for distinguishing microbial mats and earths, in Noffke, N., Chafetz, H. (eds.), Microbial mats in siliciclastic depositional systems through time: Tulsa, Oklahoma, USA, SEPM Special Publication 101, 139-152.

Retallack, G.J., 2013, Ediacaran life on land: Nature, 493, 89-92.

Retallack, G.J., Mindszenty, A., 1994, Well preserved Late Precambrian paleosols from northwest Scotland: Journal of Sedimentary Research, A64, 264-281.

Reynolds, R., Belnap, J., Reheis, M., Lamothe, P., Luiszer, F., 2001 Aeolian dust in Colorado Plateau soils: nutrient inputs and recent change in source: Proceedings of the National Academy of Sciences USA, 98, 7123-7127.

Rino, S., Komiya, T., Windley, B.F., Katayama, S., Motoki, A., Hirata, T., 2004, Major episodic increases of continental crust growth determined from zircon ages of river sands; implication for mantle overturns in the early Precambrian: Physics of the Earth and Planetary Interiors, 146, 369-394.

Romans, B.W., Graham, S.A., 2013, A Deep-Time Perspective of LandOcean Linkages in the Sedimentary Record: Annual Review of Marine Sciences, 5, 69-94

Rosing, M.T., Frei, R., 2004, U-rich Archaean sea-fl oor sediments from Greenland: indications of $>3700 \mathrm{Ma}$ oxygenic photosynthesis: Earth and Planetary Science Letters, 217, 237-244.

Rosing, M.T., Bird, D.K., Sleep, N.H., Glassley, W., Albarede, F., 2006, The rise of continents - An essay on the geologic consequences of photosynthesis: Palaeogeography, Palaeoclimatology, Palaeoecology, 232, 99-113.

Rossi, F., Potrafka, R.M., Garcia-Pichel, F., De Philippis, R., 2012, The role of the exopolysaccharides in enhancing hydraulic conductivity of biological soil crusts: Soil Biology and Biochemistry, 46, 33-40.

Rye, R., Holland, H.D., 2000, Life associated with a 2.76 Ga ephemeral pond? Evidence from Mount Roe \#2 paleosol: Geology, 28, 483-486.

Santosh, M., 2010, A synopsis of recent conceptual models on supercontinent tectonics in relation to mantle dynamics, life evolution and surface environment: Journal of Geodynamics, 50, $116-133$.

Sarbu, S.M., Kane, T.C., Kinkle, B.K., 1996, A chemoautotrophically based cave ecosystem: Science, 272, 1953-1955.

Schieber, J., Bose, P.K., Eriksson, P.G., Banerjee, S., Sarkar, S., Altermann, W., Catuneau, O. (Eds.), 2007, Atlas of Microbial Mat Features Preserved within the Clastic Rock Record: Amsterdam, The Netherlands, Elsevier, 324 p.

Schiffbauer, J.D., Xiao, S., 2009, Novel application of Focused Ion Beam-Electron Microscopy (FIB-EM) in preparation and analysis of microfossils ultrastructures: a new view of complexity in early eukaryotic organisms: PALAIOS, 24, 616-626.

Schiffbauer, J.D., Yin, L., Bodnar, R.J., Kaufman, A.J., Meng, F., Hu, J., Shen, B., Yuan, X., Bao, H., Xiao, S., 2007, Ultrastructural and geochemical characterization of Archean-Paleoproterozoic graphite particles: implications for recognizing traces of life in highly metamorphosed rocks: Astrobiology, 7, 684-704.

Schiffbauer, J.D., Wallace, A.F., Hunter, J.L. Jr., Kowalewski, M., Bodnar, R.J., Xiao, S., 2012, Thermally-induced structural and chemical alteration of organic-walled microfossils: an experimental approach to understanding fossil preservation in metasediments: Geobiology, $10,402-423$.

Schirrmeister, B.E., de Vosb, J.M., Antonelli, A., Bagheri, H.C., 2013, Evolution of multicellularity coincided with increased diversification of cyanobacteria and the Great Oxidation Event: Proceedings of the National Academy of Sciences USA, 110, 1791-1796.

Schmidt, S.K., Reed, S.C., Nemergut, D.R., Stuart-Grandy, A., Cleveland, C.C., Weintraub, M.N., Hill, A.W., Costello, E.K., Meyer, A.F., Neff, J.C., Martin, A.M., 2008, The earliest stages of ecosystem succession in high-elevation ( 5000 metres above sea level), recently deglaciated soils: Proceedings of Biological Sciences, 275, 2793-2802.

Schopf, W.J., 1968, Microflora of the Bitter Springs Formation, late Precambrian, central Australia: Journal of Paleontology, 42, 651-688.

Schopf, J.W., 1983, Earth's Earliest Biosphere: Its Origin and Evolution: Princeton, New Jersey, USA, Princeton University Press, Princeton, $610 \mathrm{p}$.

Schopf, J.W., 1992a, Paleobiology of the Archean, in Schopf, J.W., Klein, C. (eds.), The Proterozoic Biosphere: New York, New York, USA, Cambridge University Press, 25-40. 
Schopf, J.W., 1992b, Proterozoic Prokaryotes: Affinities, Geologic Distribution, and Evolutionary trends, in Schopf, J.W., Klein, C. (eds.), The Proterozoic Biosphere: New York, New York, USA, Cambridge University Press, 497.

Schopf, J.W., Klein, C., 1992, The Proterozoic Biosphere: New York, New York, USA, Cambridge University Press, $1348 \mathrm{p}$.

Schopf, J.W., Walter, M.R., Ruiji, C., 2007, Earliest evidence of life on earth: Precambrian Research, 158, 139-140.

Schwartzman, D.W., Volk, T., 1989, Biotic Enhancement of Weathering and the Habitability of Earth: Nature, 340, 457-460.

Shear, W.A., 1991, The early development of terrestrial ecosystems: Nature, 351, 283-289.

Sheldon, N.D., 2012, Microbially Induced Sedimentary Structures in the ca. 1100 Ma terrestrial midcontinent rift of North America, in Noffke, N., Chafetz, H. (eds.), Microbial mats in siliciclastic depositional systems through time: Tulsa, Oklahoma, USA, SEPM Special Pub. 153-162.

Shen, Y., Buick, R., 2004, The antiquity of microbial sulfate reduction: Earth Science Reviews, 64, 243-272.

Shen, Y., Farquhar, J., Masterson, A., Kaufman, A.J., Buick, R., 2009, Evaluating the role of microbial sulfate reduction in the early Archean using quadruple isotope systematics: Earth and Planetary Science Letters, 279, 383-391.

Shephard, K.L., 1987, Evaporation of water from the mucilage of a gelatinous algal community: British Phycological Journal, 22, 181-185.

Sigler, W.V., Crivii, S., Zeyer, J., 2002, Bacterial succession in glacial forefield soils characterized by community structure, activity and opportunistic growth dynamics: Microbiology Ecology, 44, 306-316.

Simpson, W.S., Simpson, E.L., Wizevich, M.C., Malenda, H.F., HilbertWolf, H.L., Tindall, S.E., 2010, A preserved Late Cretaceous biological soil crust in the capping sandstone member, Wahweap Formation, Grand Staircase-Escalante National Monument, Utah, Palaeoclimatic implications: Sedimentary Geology, 230, 139-145.

Simpson, E.L., Heness, E.A., Bumby, A.J., Eriksson, P.G., Eriksson, K.A., Hilbert-Wolf, H.A., Linnevelt, S., Malenda, H.F., Modungwa, T., Okafor, O.J., 2013, Evidence for $2.0 \mathrm{Ga}$ continental microbial mats in a paleodesert setting': Precambrian Research, 237(10), 36-50.

Singh, S.P., Kumari, S., Rastogi, R.P., Singh, K.L., Singha, R., Singha, R.P., 2010, Photoprotective and biotechnological potentials of cyanobacterial sheath pigment, scytonemin: African Journal Biotechnology, 9, 580-588.

Sinha, R.P., Häder, D.P., 2002, UV-induced DNA damage and repair: a review: Photochemical Photobiology Sciences, 1, 225-236.

Skotnicki, S.J., Knauth, L.P., 2007, The Middle Proterozoic Mescal Paleokarst, Central Arizona, USA: karst development, silicification, and cave deposits: Journal of Sedimentary Research, 77, 1046-1062

Sleep, N.H., Bird, D.K., 2007, Niches of the pre-photosynthetic biosphere and geologic preservation of Earth's earliest ecology: Geobiology, $5,101-117$.

Smith, S.M., Abed, R.M.M., Garcia-Pichel, F., 2004, Biological soil crusts of sand dunes in Cape Cod National Seashore, Massachusetts, USA: Microbial Ecology, 48, 200-208.

Smith, S.E., Read, D., 2008, Mycorrhizal Symbiosis ( $3^{\text {rd }}$ Edition): New York, New York, USA, Elsevier Ltd, 800 p.

Squire, R.J., Stewart, I.R., Zang, W.L., 2006, Acritarchs in polydeformed and highly altered Cambrian rocks in western Victoria: Australian Journal of Earth Sciences, 53, 697-705.

Stern, R.J., Scholl, D.W., 2010, Yin and yang of continental crust creation and destruction by plate tectonic processes: International Geology Review, 52, 1-31.

Strother, P.K., Battison, L., Brasier, M.D., Wellman, C.H., 2011, Earth's earliest non-marine eukaryotes: Nature, 473, 505-509.

Stüeken, E.E., Catling, D.C., Buick, R., 2012, Contributions to late Archaean sulphur cycling by life on land: Nature Geoscience, 5, 722-725.

Summons, R.E., Jahnke, L.L., Hope, J.M., Logan, G.A., 1999, 2-Methylhopanoids as biomarkers for cyanobacterial oxygenic photosynthesis: Nature, 400, 554-557.

Sun, H.J., Friedmann, E.I., 1999, Growth on geological time scales in the Antarctic cryptoendolithic microbial community: Geomicrobiology Journal, 16, 193-202.

Takeuchi, N., Kohshima, S., Seko, K., 2001, Structure, formation, darkening process of albedo reducing material (cryoconite) on a Himalayan glacier: a granular algal mat growing on the glacier: Arctic, Antarctic, and Alpine Research, 33, 115-122.

Taylor, T.N., Taylor, E.L., Krings, M., 2009, Paleobotany, The Biology and Evolution of Fossil Plants: Amsterdam, The Netherlands, Elsevier, $1252 \mathrm{p}$.

Thiry, M., Simon-Coincon, R., 1999, Palaeoweathering, Palaeosurfaces and Related Continental Deposits: Oxford, United Kingdom, Blackwell Science Ltd. 408 p.

Tice, M.M., Lowe, D.R., 2004, Photosynthetic microbial mats in the 3,416-Myr-old ocean: Nature, 431, 549-552.

Tirkey, J., Adhikary, S.P., 2005, Cyanobacteria in the Biological soil crusts of India: Current Science, 89, 515-521.

Tomitani, A., Knoll, A.H., Cavanaugh, C.M., Ohno, T., 2006, The evolutionary diversification of cyanobacteria: molecularphylogenetic and paleontological perspectives: Proceedings of the National Academy of Sciences USA, 103, 5442-5447.

Trendall, A.F., Blockley, J.G., 2004, Precambrian iron-formations, in Eriksson, P.G., Altermann, W., Nelson, D.R., Mueller, W.U., Catuneanu, O. (eds.), The Precambrian Earth: Tempos and Events, Developments in Precambrian Geology 12: Amsterdam, The Netherlands, Elsevier, 403-421.

Ueno, Y., Yamada, K., Yoshida, N., Maruyama, S., Isozaki, Y., 2006, Evidence from fluid inclusions for microbial methanogenesis in the early Archaean era: Nature, 440, 516-519.

van Kranendonk, M.J., 2004, Archaean Tectonics 2004, A review: Precambrian Research, 131, 143-151.

van Kranendonk, M.J., 2011, Morphology as an indicator of biogenicity for Earth's oldest fossils from the 3.5-3.4 Ga Pilbara Craton, Western Australia, in Reitner, J., Queric, N.V., Arp, G. (eds.), Advances in Stromatolite Geobiology. Lecture Notes in Earth Sciences 131,: Berlin, Germany, Springer, 517-534.

van Kranendonk, M.J., Smithies, R.H., Bennett, V., 2007, Earth's Oldest Rocks. Developments in Precambrian Geology, Series 15: Amsterdam, The Netherlands, Elsevier, $466 \mathrm{p}$.

van Kranendonk, M.J., Philippot, P., Lepot, K., Bodorkos, S., Pirajno, F., 2008, Geological setting of Earth's oldest fossils in the c. $3.5 \mathrm{Ga}$ Dresser Formation, Pilbara Craton, Western Australia: Precambrian Research, 167, 93-124.

van Zuilen, M.A., Lepland, A., Arrhenius, G., 2002, Reassessing the evidence for the earliest traces of life: Nature, 418, 627-630.

Voigt, E., 1972, Tonrollen als potentielle pseudofossilien : Natur und Museum, 102, 401-410.

Wacey, D., Kilburn, M.R., Saunders, M., Cliff, J., Brasier, M.D., 2011, Microfossils of sulphur-metabolizing cells in 3.4-billion-year-old rocks of Western Australia: Nature Geoscience, 4, 698-702.

Walsh, M.M., 1992, Microfossils and possible microfossils from the Early Archean Onverwacht Group, Barberton Mountain Land, South Africa: Precambrian Research, 54, 271-292.

Walsh, M.M., Lowe, D.R., 1985, Filamentous microfossils from the 3500 Myr-old Onverwacht Group, Barberton Mountain Land, South Africa: Nature, 314, 530-532.

Walter, M.R., 1983, Archean stromatolites: evidence of the Earth's earliest benthos, in Schopf, J.W. (ed.) Earth's Earliest Biosphere: Princeton, New Jersey, USA, Princeton University Press, 187-213.

Walter, M.R., Buick, R., Dunlop, J.S.R., 1980, Stromatolites 3,400-3,500 Myr old from the North Pole area, Western Australia: Nature, 284, 443-445.

Watanabe, Y., Martini, J.E.J., Ohmoto, H., 2000, Geochemical evidence for terrestrial ecosystems 2.6 billion years ago: Nature, 408, 574-578.

Welch, S.A., Barker, W.W., Banfield, J.F., 1999, Microbial extracellular polysaccharides and plagioclase dissolution: Geochimica et Cosmochimica Acta, 63, 1405-1419. 
Westall, F., 2010, Early life: nature, distribution and evolution, in Gargaud, M., López-Garcìa, P., Martin, H. (eds.), Origins and Evolution of Life. An Astrobiological Perspective: Cambridge, Massachusetts, USA, Cambridge University Press, 391-413.

Westall, F., De Wit, M.J., Dann, J., Van Der Gaast, S., De Ronde, C., Gerneke, D., 2001, Early Archaean fossil bacteria and biofilms in hydrothermally influenced, shallow water sediments, Barberton Greenstone Belt, South Africa: Precambrian Research, 106, 91-112.

Westall, F., de Vries, S.T., Nijman, W., Rouchon, V., Orberger, B., Pearson, V., Watson, J., Verchovsky, A., Wright, I., Rouzaud, J.N., Marchesini, D., Anne, S., 2006a, The 3.466 Ga Kitty's Gap Chert, an Early Archaean microbial ecosystem, in Reimold W.U. and Gibson, R. (ed.), Processes on the Early Earth: Boulder, Colorado, USA, Geological Society of America Special Paper, 405, 105-131.

Westall, F., de Ronde, C.E.J., Southam, G., Grassineau, N., Colas, M., Cockell, C., Lammer, H., 2006b, Implications of a 3.472-3.333 Ga-old subaerial microbial mat from the Barberton Greenstone Belt, South Africa for the UV environmental conditions on the early Earth: Philosophical Transactions of the Royal Society of London, B Biology, 361, 1857-1875.

Westall, F., Cavalazzi, B., Lemelle, L., Marrocchi, Y., Rouzaud, J.N., Simionovici, A., Salomé, M., Mostefaoui, S., Andreazza, C., Foucher, F., Toporski, J., Jauss, A., Thiel, V., Southam, G., MacLean, L., Wirick, S., Hofmann, A., Meibom, A., Robert, F., Défarge, C., 2011, Implications of in situ calcification for photosynthesis in a 3.3 Ga-old microbial biofilm from the Barberton greenstone belt, South Africa: Earth and Planetary Science Letters, 310, 468-479.

White, D., 2000, The Physiology and Biochemistry of Prokaryotes, 2nd Ed.: Oxford, United Kingdom, Oxford University Press, 565 p.

Whitton, B.A., Potts, M., 2000, The Ecology of Cyanobacteria: Their Diversity in Time and Space: Dordrecht, The Netherlands, Kluwer Academic Publisher, $669 \mathrm{p}$.

Wilde, S.A., Valley, J.W., Peck, W.H., Graham, C.M., 2001, Evidence from detrital zircons for the existence of continental crust and oceans on the Earth 4.4 Gyr ago: Nature, 409, 175-178.

Williams, J.D., Dobrowolsk, J.P., West, N.E., Gillette, D.A., 1995, Microphytic crust influence on wind erosion: Transactions of the ASAE, 38, 131-137.

Williams, A.J., Buck, B.J., Beyene, M.A., 2012, Biological Soil Crusts in the Mojave Desert, USA: Micromorphology and Pedogenesis: Soil Science Society of America Journal, 76, 1685-1695.

Windley, B., 2007, Overview and history of investigation of early Earth rocks, in van Kranendonk, M.J., Smithies, R.H., Bennett, V. (eds.), Earth's Oldest Rocks. Developments in Precambrian Geology, Series 15: Amsterdam, The Netherlands Elsevier, 3-7.

Wright, V.P., 1985, The precursor environment for vascular plant colonization: Philosophical Transactions of the Royal Society of London, B Biology, 309, 143-145.
Xiao, S., Kaufman, A.J., 2006, Neoproterozoic Geobiology and Paleobiology. Topics in Geobiology 27: Dordrecht, The Netherlands, Springer, $300 \mathrm{p}$.

Xiao, S., Knauth, L.P., 2013, Palaeontology: Fossils come in to land: Nature, 493, 28-29.

Xiong, J., Fischer, W.M., Inoue, K., Nakahara, M., Bauer, C.E., 2000, Molecular evidence for the early evolution of photosynthesis: Science, 289, 1724-1730.

Yang, W., Holland, H.D., 2003, The Hekpoort paleosol in strata 1 at Garborone, Botswana: soil formation during the Great Oxidation Event: American Journal of Science, 303, 187-220.

Yasui, A., McCready, S.J., 1998, Alternative repair pathways for UVinduced DNA damage: Bioessays, 20, 291-297.

Yu, G., Jacobsen, S.B., 2011, Fast accretion of the Earth with a late Moonforming giant impact: Proceedings of the National Academy of Sciences USA, 108, 17604-17609.

Zang, W.L., 2007, Deposition and deformation of late Archean sediments and preservation of microfossils in the Harris Greenstone Domain, Gawler Craton, South Australia: Precambrian Research, 156, 107-124.

Zbinden, E.A., Holland, H.D., Feakes, C.R., Dobos, S.K., 1988, The Sturgeon Falls paleosol and the composition of the atmosphere 1.1 Ga BP: Precambrian Research, 42, 141-163.

Zhang, J., 1992, Observation on Algal Effects on Subaerial Karst Sedimentation: Geographical Research, 11 (2), 26-33.

Zhao, B., Robb, L.J., Harris, C., Jordaan, L.J., 2006, Origin of hydrothermal fluids and gold mineralization associated with the Ventersdorp contact reef, Witwatersrand Basin, South Africa: Constraints from S, $\mathrm{O}$, and $\mathrm{H}$ isotopes, in Reimold, W.U., Gibson, R.L. (Eds.), Processes on the Early Earth. GSA Special Paper, 405, 333-352.

Zhuravlev, A.Y., Riding, R., 2001, The Ecology of the Cambrian radiation: New York, New York, USA, Columbia University Press, 525 p.

Zonneveld, K.A.F., Versteegh, G.J.M., Kasten, S., Eglinton, T.I., Emeis, K.C., Huguet, C., Koch, B.P., De Lange, G.J., De Leeuw, J.W., Middelburg, J.J., Mollenhauer, G., Prahl, F.G., Rethemeyer, J., Wakeham, S.G., 2010, Selective preservation of organic matter in marine environments; processes and impact on the sedimentary record: Biogeosciences, 7, 483-511.

Manuscrito recibido: Octubre 31, 2013.

Manuscrito aceptado: Noviembre 1, 2013. 